\title{
Article
}

\section{Dark Matter as a Metric Perturbation}

\author{
W. M. Stuckey ${ }^{1 *}$, Timothy McDevitt ${ }^{2}$, A. K. Sten ${ }^{1}$ and Michael Silberstein ${ }^{3,4}$ \\ Department of Physics, Elizabethtown College, Elizabethtown, PA 17022, USA \\ Department of Mathematics, Elizabethtown College, Elizabethtown, PA 17022, USA \\ Department of Philosophy, Elizabethtown College, Elizabethtown, PA 17022, USA \\ 4 Department of Philosophy, University of Maryland, College Park, MD 20742, USA \\ * Correspondence: stuckeym@etown.edu; Tel.: +1-717-361-1436
}

\begin{abstract}
Since general relativity (GR) has already established that matter can simultaneously have two different values of mass depending on its context, we argue that the missing mass attributed to non-baryonic dark matter (DM) actually obtains because there are two different values of mass for the baryonic matter involved. The globally obtained "dynamical mass" of baryonic matter can be understood as a small perturbation to a background spacetime metric even though it's much larger than the locally obtained "proper mass." Having successfully fit the SCP Union2.1 SN Ia data without accelerating expansion or a cosmological constant, we employ the same ansatz to compute dynamical mass from proper mass and explain galactic rotation curves (THINGS data), the mass profiles of X-ray clusters (ROSAT and ASCA data) and the angular power spectrum of the cosmic microwave background (Planck 2015 data) without DM. We compare our fits to modified Newtonian dynamics (MOND), metric skew-tensor gravity (MSTG) and scalar-tensor-vector gravity (STVG) for each data set, respectively, since these modified gravity programs are known to generate good fits to these data. Overall, we find our fits to be comparable to those of MOND, MSTG and STVG. While this favorable comparison does not establish the validity of our proposition, it does provide confidence in using the fits to pursue an underlying action. Indeed, the functional form of our ansatz reveals an interesting structure in these fits.
\end{abstract}

Keywords: dark matter; THINGS; MOND; metric skew-tensor gravity; scalar-tensor-vector gravity; $\mathrm{CMB}$ angular power spectrum

PACS: 95.35.+d; 95.30.Sf; 04.90.+e

\section{Introduction}

Since the early 1930's, galactic rotation curves (RC's) and galactic cluster masses have been known to deviate from Newtonian expectations based on luminous matter and known mass-to-luminosity ratios [1-4]. These are two aspects of the dark matter problem [5], making this one of the most persistent problems in physics. As a consequence, many approaches have been brought to bear on the dark matter problem, typically by way of new particles [6,7], but also by way of modifications to existing theories of gravity, e.g., modified Newtonian dynamics (MOND) [8-10] and relativistic counterparts [11-15], Moffat's modified gravity (MOG), i.e., metric skew-tensor gravity (MSTG) and scalar-tensor-vector gravity (STVG) [16-23], Renormalization Group corrected General Relativity [24], and nonlocal general relativity [25]. No one disputes the existence of baryonic dark matter, e.g., brown dwarfs, black holes, and molecular hydrogen, but there is wide agreement that baryonic dark matter does not exist in large enough supply to resolve the dark matter problem [26]. While the assumption of non-baryonic dark matter (DM) in $\Lambda$ CDM cosmology works well for explaining cosmological features (scales greater than $1 \mathrm{Mpc}$ ), there is still no independent verification of non-baryonic dark matter [27], galactic RC's do not conform to the theoretical predictions of $\Lambda \mathrm{CDM}$ for the distribution of DM on galactic scales [28-31] and there are five seemingly incompatible properties that must be satisfied by DM, i.e., dark, cold, abundant, stable, and dissipationless [32]. "As Tom Shanks once said, there are only two things wrong with $\Lambda$ CDM: $\Lambda$ and CDM" [33]. 
Therefore, it is reasonable at this stage to consider modifications to general relativity (GR) that deny the need for DM in resolving the dark matter problem.

Accordingly, we explain galactic RC's, the mass profiles of X-ray clusters, and the angular power spectrum of the cosmic microwave background (CMB) without DM based on the baryonic matter involved simultaneously having two different values of mass as occurs in general relativity (GR). For example, when a Schwarzschild vacuum surrounds a spherical matter distribution the "proper mass" $M_{p}$ of the matter (the mass measured locally in the matter) can be different than the "dynamical mass" $M$ in the Schwarzschild metric responsible for orbital kinematics about the matter [34, p. 126]. Likewise, suppose a Schwarzschild vacuum surrounds a sphere of Friedmann-Lemaitre-Robertson-Walker (FLRW) dust connected at the instanteously null Schwarzschild radial coordinate. The dynamical mass $M$ of the surrounding Schwarzschild metric is related to the proper mass $M_{p}$ of the FLRW dust by [35]

$$
\frac{M_{p}}{M}= \begin{cases}1 & \text { flat } \\ \frac{3(\eta-\sin (\eta))}{4 \sin ^{3}(\eta / 2)} \geq 1 & \text { positively curved } \\ \frac{\left.3\left(\sinh ^{3} \eta\right)-\eta\right)}{4 \sinh ^{3}(\eta / 2)} \leq 1 & \text { negatively curved }\end{cases}
$$

As one possible justification for this approach, the two values of mass might be understood to result from "disordered locality" [36,37], i.e., allowing the links of a Regge calculus graph [38-41] to connect non-neighboring points of the corresponding smooth GR spacetime manifold M. Disordered locality might result, for example, from direct action [42-47] which modifies lattice gauge theory per large graphical links. Essentially, if direct action is correct, then photon exchanges on astronomical scales will introduce disordered locality that can certainly be expected to perturb the corresponding local, background GR spacetime geometry. In this graphical view, it is easy to depict the two different values of mass for the same matter by associating matter with nodes and its mass with links. Links to neighboring nodes (in the context of $\mathrm{M}$ ) would harbor proper mass while links to non-neighboring nodes (disordered locality) would harbor dynamical mass. Other justifications surely exist, but we will refrain from further theoretical speculation here.

While dynamical mass can be $\sim 10$ times larger than proper mass, the correction to the background spacetime metric responsible for this difference is $\ll 1$, as we show in section 2 . Thus, what we're advocating is a geometric view of even weak gravitational fields. Indeed, Cooperstock et al. used GR instead of Newtonian gravity in fitting galactic RC's and found that the non-luminous matter in galaxies "is considerably more modest in extent than the DM extent claimed on the basis of Newtonian gravitational dynamics" [48-50]. And, Moffat \& Rahvar used "The weak field approximation of MOG" as a perturbation of "the metric and the fields around Minkowski space-time" in fitting galactic RC's [21] and X-ray cluster mass profiles [22] without DM. Certainly MOND can be viewed in this fashion, since MOND advocates an extremely small modification to acceleration on astronomical scales in the context of flat spacetime (Newtonian gravity), and acceleration due to gravity in flat spacetime is replaced by curved spacetime in GR. So, MOND could be viewed as saying that a small change in spacetime curvature (equivalent to a small change to acceleration in Newtonian gravity) replaces the need for a greatly increased mass in accounting for galactic dynamics. In fact, McGaugh et al. found [51] "a strong relation between the observed radial acceleration $g_{\text {obs }}$ and that due to the baryons, $g_{b a r}$ " which "suggests that the baryons are the source of the gravitational potential." Thus, MOND could be interpreted as saying precisely what we're claiming, i.e., small spacetime curvature perturbations on astronomical scales can account for dark matter phenomena without DM.

We previously generated an ansatz to fit the SCP Union2/2.1 SN Ia data matching that of $\Lambda \mathrm{CDM}$ via a simple perturbative geometric correction of proper distance in Einstein-deSitter (EdS) 
cosmology [52-54]. The resulting explanation did not harbor accelerating expansion, so there was no need of a cosmological constant or dark energy. Similarly here, we use this ansatz to replace DM in fitting galactic RC's (section 3), the mass profiles of X-ray clusters (section 4), and the angular power spectrum of the $\mathrm{CMB}$ (section 5). As it turns out, the functional form of the ansatz reveals an interesting structure in these fits that may provide clues to an underlying action.

\section{The Ansatz}

In the case of dark energy as pertains to the SCP Union2.1 SN Ia data, we considered corrections $h_{\alpha \beta}$ satisfying the vacuum perturbation equation $\nabla^{2} h_{\alpha \beta}=0$ in the flat space of EdS cosmology, i.e., $\frac{d^{2}}{d D_{p}^{2}} h_{i i}=0$ where $D_{p}$ is proper distance per the EdS metric. We then corrected proper distance according to $D_{p} \rightarrow \sqrt{1+h_{i i}} D_{p}=\sqrt{1+\frac{D_{p}}{A}} D_{p}$ ( $A$ is an arbitrary constant used as a fitting parameter), which is the general form of a metric dot product used to produce a vector magnitude. Here we generalize this approach via the following ansatz ${ }^{1}$

If the quantity $X$ in the context of the corresponding GR spacetime requires modification due to disordered locality, then $X \rightarrow \sqrt{1+h} X$ where $h$ satisfies the standard vacuum perturbation equation of GR and exploits a symmetry of the GR spacetime.

The symmetry in the dark energy application was the flat space of EdS. Here, for galactic rotation curves, X-ray cluster mass profiles, and the baryon-photon perturbations in pre-recombination FLRW cosmology, we will assume spherical symmetry so that $h$ satisfies $\nabla^{2} h(r)=0$ in spherical coordinates. It does not have to be the case that $h \ll 1$, i.e., $h$ does not have to be a small change, as is obviously the case with DM phenomena where dynamical mass can be $\sim 10$ times as large as proper mass. However, as we will show, in all three cases the total perturbative correction to the background spacetime geometry obtained after adjusting proper mass to dynamical mass per the ansatz is still $\ll 1$. In all three cases, we are modifying what is already a weak term in the Schwarzschild metric, so we are essentially correcting a Newtonian potential (first-order Schwarzschild metric). In the FLRW case, where the background FLRW metric being corrected is truly relativistic, we must specify the gauge. However, as we will see in section 5, there is a Newtonian gauge for the perturbations that accommodates this view.

We should point out that even the relativistic EdS solution allows for a Newtonian-like characterization per Regge calculus. As we obtained previously [52], the Regge equation for EdS cosmology with continuous time is

$$
\frac{\pi-\cos ^{-1}\left(\frac{v^{2} / c^{2}}{2\left(v^{2} / c^{2}+2\right)}\right)-2 \cos ^{-1}\left(\frac{\sqrt{3 v^{2} / c^{2}+4}}{2 \sqrt{v^{2} / c^{2}+2}}\right)}{\sqrt{v^{2} / c^{2}+4}}=\frac{G m}{2 r c^{2}}
$$

With $v^{2} / c^{2} \ll 1$ an expansion of the LHS of $\mathrm{Eq}(2)$ gives

$$
\frac{v^{2}}{4 c^{2}}+\mathcal{O}\left(\frac{v}{c}\right)^{4}=\frac{G m}{2 r c^{2}}
$$

Thus, to leading order (defining "small" simplices required to accurately approximate GR) we have $\frac{v^{2}}{2}=\frac{G m}{r}$, which is just a Newtonian conservation of energy expression for a unit mass moving at escape velocity $v$ at distance $r$ from mass $m$. So, in the case of Regge EdS cosmology, we see that spacetime curvature enters as a deficit angle between adjoining Newtonian simplices. We're certainly

1 This ansatz is not generally covariant, so it cannot be the final word. Our goal at this stage is to seek data fits with the ansatz that will provide clues to an underlying action. 
not advocating a Newtonian approach to gravity, quite the contrary. What we're advocating is a geometric view of even weak gravitational fields, as we pointed out in section 1.

Returing to our ansatz, the solution $h=\frac{A}{r}+B$ is then used to obtain dynamical mass $M$ from proper mass $M_{p}$ per $M=\sqrt{1+h} M_{p}=\sqrt{\frac{A}{r}+B} M_{p}$, where this geometric correction is applied to the Newtonian potential in the GR spacetime metric. As with dark energy, the arbitrary constants $A$ and $B$ are used as fitting parameters at this stage in the program. The values of these fitting parameters in galactic, galactic cluster, and cosmological contexts will then provide guides to the construction of an underlying action. Indeed, as we will see, the best fit values of $A$ and $B$ show interesting trends across the three data sets.

Since galactic RC's are expected to follow Newtonian predictions to the technical limits of current astronomical observations, we will talk unambiguously about spatial hypersurfaces in the context of the background GR spacetime structure. In the case of galactic RC's and galactic clusters, the GR background spacetime is a Schwarzschild vacuum. For cosmology, the GR background spacetime is spatially flat FLRW, but the functional form of our correction will still be $h=\frac{A}{r}+B$, since the baryon-photon perturbations are spherically symmetric. Our use of GR spacetime with perturbing Newtonian potentials is called the "Newtonian gauge" [55].

Although "The Kerr metric does not represent the exterior metric of a physically likely source, nor the metric during any realistic gravitational collapse" [56], we might nonetheless consider it structurally since rotating galaxies are prima facie axisymmetric. In Boyer-Linquist coordinates, the Kerr metric is (geometrized units)

$$
d s^{2}=-\left(1-\frac{2 M r}{\Sigma}\right) d t^{2}-\frac{4 M a r \sin ^{2} \theta}{\Sigma} d t d \phi+\frac{\Sigma}{\Delta} d r^{2}+\Sigma d \theta^{2}+r^{2}\left(1+\frac{a^{2}}{r^{2}}+\frac{2 M a^{2} \sin ^{2} \theta}{r \Sigma}\right) d \phi^{2}
$$

where $\Sigma=r^{2}+a^{2} \cos ^{2} \theta$ and $\Delta=r^{2}-2 M r+a^{2}$. This reduces to the Schwarzschild metric

$$
d s^{2}=-\left(1-\frac{2 M}{r}\right) d t^{2}+\left(1-\frac{2 M}{r}\right)^{-1} d r^{2}+r^{2} d \Omega^{2}
$$

when $a \ll r\left(a=\frac{J}{M}\right.$ is the angular momentum per unit mass). If for a given galactic orbital radius $r$ we assume all the mass $M$ interior to that radius resides in a radially and axially thin, disk-shaped annulus at $r$ with orbital velocity $v$ in order to generate the most conservative (largest) estimate of $J$, we have $a=\frac{M r^{2} \omega}{M c}=r \frac{v}{c}$ (restoring c). Since, the largest galactic orbital velocities are about $300 \mathrm{~km} / \mathrm{s}$, we have $a \sim r / 1000$ so we will use the much simpler Schwarzschild metric for our discussions. Such approximations are not unusual in astrophysics, since it is the case that "for realistic distributions of matter in galaxies, we have neither analytic, nor numerical solutions to general relativity from which orbits can be predicted" [57].

We now show that small differences in spacetime geometry can be associated with relatively large differences in the values of mass. For example, while $M$ can be $\sim 10 M_{p}$, the deviation from flat spacetime on typical galactic scales per $\frac{2 G M(r)}{c^{2} r}$ of the Schwarzschild metric is negligible given current technical limits on astronomical observations. Assuming circular orbits (which is common for fitting galactic RC's) we have $v^{2} r=G M(r)$, where $M(r)$ is the dynamical mass inside the circular orbit at radius $r$ and $v$ is the orbital speed. This gives $\frac{2 G M(r)}{c^{2} r}=2 \frac{v^{2}}{c^{2}}$. Again, the largest galactic rotation speeds are typically only $10^{-3} \mathrm{c}$ so the metric deviation from flat spacetime to include the larger dynamical mass is $\sim 10^{-6}$, an empirically small metric correction.

Another way to quantify the difference geometrically between $M$ and $M_{p}$ is to use ${ }^{(3)} R=\frac{16 \pi G \rho}{c^{2}}$ [58] on galactic scales (where $\rho$ is the mass density). Assuming constant mass density (typical in a 
galactic bulge) out to radius $r$ where we have orbital velocity $v$, we have $\frac{16 \pi G \rho}{c^{2}}=\frac{12 v^{2}}{r^{2} c^{2}}$. Constant density means $M(r) \sim r^{3}$ so

$$
v(r)=\sqrt{\frac{G M(r)}{r}}
$$

increases linearly with distance from zero to its max value $v_{\max }$. Assuming this happens at the radius of the bulge $r_{b}$ (galactic RC's actually peak farther out, so this overestimates the effect), we have ${ }^{(3)} R=$ $\frac{12 v_{\max }^{2}}{r_{b}^{2} c^{2}}$ for the spatial curvature scalar in the annulus at $r$ rather than its Schwarzschild value of zero. $v_{\text {max }} \approx 300 \mathrm{~km} / \mathrm{s}$ and $r_{b} \approx 2000 \mathrm{pc}$ gives ${ }^{(3)} R \sim 10^{-45} / \mathrm{m}^{2}$. So, a factor of ten one way or the other in $\rho$ is geometrically inconsequential in this context. Intracluster medium gas is even more rarefied and the potentials used for FLRW metric perturbations leading to CMB anisotropies are already $\ll 1$ to include DM. For all these reasons, we believe it's sensible to speculate on a perturbative correction to GR spacetime geometry that increases the baryonic mass to account for DM phenomena.

\section{Galactic Rotation Curves}

In the MOND approach with spherical symmetry and circular orbits, as is commonly used for fitting galactic $R C^{\prime}$ s, it is the Newtonian acceleration

$$
a_{N}(r)=\frac{G M(r)}{r^{2}}
$$

that is modified by the so-called interpolating function $\mu(x)$ where $x=\left|\frac{a(r)}{a_{0}}\right|$ with $a_{0}$ a universal constant so that

$$
\mu(x) a(r)=\frac{G M(r)}{r^{2}}
$$

$\mu(x) \sim x$ for $x \ll 1$ and $\mu(x) \sim 1$ for $x \gg 1$. Of course, $\mu(x(r))$ means one could view $\mathrm{Eq}(8)$ as a modification to $M(r)$ or $G$ as in MSTG [57]

$$
a(r)=\frac{G M(r)}{\mu(r) r^{2}}
$$

where $G(r)=\frac{G}{\mu(r)}$ in MSTG. In STVG in $k$ space we also have $G \rightarrow G_{e f f}$, as we will see in section 5 . In terms of the Newtonian potential $\chi$, the Schwarzschild metric is

$$
d s^{2}=-c^{2}\left(1+\frac{2 \chi}{c^{2}}\right) d t^{2}+\left(1+\frac{2 \chi}{c^{2}}\right)^{-1} d r^{2}+r^{2} d \Omega^{2}
$$

where $a(r)=|\nabla(\chi)|$. So, in that sense our ansatz, MOND, MSTG, and STVG can all be viewed as MOG [57]. Therefore, our fits and MOND fits for THINGS data need not be viewed as evidence for any particular theoretical justification. Applications beyond astrophysics and cosmology will ultimately adjudicate between competing theoretical justifications. Here, we seek only to test our ansatz in fitting galactic RC's by comparing it to MOND using data where MOND fits were deemed "very successful" [59].

In the MOND fits, we will use the so-called "simple" $\mu$ function

$$
\mu(x)=\frac{x}{1+x}
$$

which Gentile et al. found most successful with the THINGS data [59]. We will also adopt their median best-fit value of the acceleration parameter for the simple $\mu$ function, $a_{0}=(1.22 \pm 0.33)$ $\mathrm{x} 10^{-8} \mathrm{~cm} \mathrm{~s}^{-2}$. Generally speaking, MOND has a single fit parameter, $M / L$ for the stellar disk, 
although when bulge data are available independent of the stellar disk, an additional $M / L$ factor can be introduced for the bulge as well. We will also allow galactic distance $d$ to vary up to one sigma from the nominal value in our MOND fits. Gentile et al. called this " $d$ constrained" [59]. Finally, Gentile et al. also note these data are "not obviously dominated by non-circular motions," so we assume circular orbits to obtain the MOND $v$ as related to the Newtonian $v_{N}[60]$

$$
v^{2}=\frac{v_{N}^{2}}{2}+\sqrt{\frac{v_{N}^{4}}{4}+v_{N}^{2} a_{0} r}
$$

where

$$
v_{N}^{2}=v_{g a s}^{2}+v_{\text {disk }}^{2}+v_{\text {bulge }}^{2}
$$

Allowing for variation in $M / L$ for the stellar disk and bulge means

$$
v_{N}^{2}=v_{\text {gas }}^{2}+v_{\text {disk }}^{2} \gamma_{\text {disk }}+v_{\text {bulge }}^{2} \gamma_{b u l g e}
$$

where $\gamma_{\text {disk }}$ and $\gamma_{\text {bulge }}$ are fit parameters. Assuming the distance $d$ to the galaxy can vary means a further correction in $M / L$ (fitting parameter) for all components, call it $\gamma_{\text {dist }}$, giving

$$
v_{N}^{2}=\left(v_{\text {gas }}^{2}+v_{\text {disk }}^{2} \gamma_{\text {disk }}+v_{\text {bulge }}^{2} \gamma_{b u l g e}\right) \gamma_{\text {dist }}
$$

where $r \rightarrow \gamma_{\text {dist }} r$ in $\mathrm{Eq}(12)$ as well. $\mathrm{Eq}(12)$ is then used to fit THINGS data, i.e., $v(r), v_{\text {gas }}(r), v_{\text {bulge }}(r)$, and $v_{\text {disk }}(r)$, using the fit parameters $\gamma_{\text {disk }}, \gamma_{g a s}$, and $\gamma_{\text {dist }}$.

Our ansatz computes the baryonic proper mass $M_{p_{i}}(r)$ for each component $i$ using Eq(6) with $v_{i}(r)$ supplied by THINGS. Then $d M_{p_{i}}=M_{p_{i}}\left(r_{2}\right)-M_{p_{i}}\left(r_{1}\right)$ for the proper mass of the $i^{t h}$ component in an annulus. The geometric modification of the ansatz is applied to each $d M_{p_{i}}$ according to its radial location to obtain the corresponding dynamical mass $d M_{i}$

$$
d M_{i}=\sqrt{\frac{A_{i}}{r}+B} d M_{p_{i}}
$$

where $A_{i}$ is a fitting factor for the $i^{\text {th }}$ component and $B$ is a fitting parameter that is the same for all components. Ultimately, we expect $A$ and $B$ to be determined from an underlying action. Thus, as we stated supra, the fitting procedures needed to resolve the problems of dark energy and dark matter should give us a hint as to the corresponding underlying action. The $d M_{i}$ are summed to produce $M_{i}(r)$, which are then summed to produce $M(r)$ which gives $v(r)$ per $\mathrm{Eq}(6)$.

We are now ready to compare our fits with MOND fits of twelve high-resolution galactic RC's from The HI Nearby Galaxy Survey [61] used by Gentile et al. [59] to explore MOND fits. [See Moffat \& Rahvar [21] for MOG fits of THINGS data.] Gentile et al. describe these data as "the most reliable for mass modelling, and they are the highest quality RC's currently available for a sample of galaxies spanning a wide range of luminosities." Keep in mind that MOND fits are more rigorously constrained, since MOND is a highly developed theory. Our fits on the other hand are being done to determine feasibility and guidance in constructing an underlying action. This is akin to allowing the acceleration parameter $a_{0}$ to vary in MOND fits so as to determine its most accurate value as a universal constant. Our results are in Table 1 and MOND results are in Table 2. Graphical display of the best fits reported below are in Figures $3 \& 4$. 
Table 1. Our fits for THINGS. MSE is in $(\mathrm{km} / \mathrm{s})^{2}$. Corresponding plots are in Figures 3 \& 4 .

\begin{tabular}{c|ccccc} 
Name & $A_{\text {bulge }}$ & $A_{\text {disk }}$ & $A_{\text {gas }}$ & $B$ & MSE \\
\hline NGC 2841 & 0.00 & 19.8 & 17600 & 0.00 & 106 \\
\hline NGC 7331 & 0.00 & 0.00 & 645 & 0.67 & 63.5 \\
\hline NGC 3521 & N/A & 0.00 & 442 & 0.67 & 243 \\
\hline NGC 6946 & 0.00 & 0.00 & 708 & 0.66 & 59.8 \\
\hline NGC 2903 & 0.00 & 18.5 & 3730 & 0.00 & 489 \\
\hline NGC 5055 & 0.00 & 1.23 & 2200 & 0.03 & 99.3 \\
\hline NGC 3198 & 0.00 & 8.33 & 705 & 0.014 & 10.1 \\
\hline NGC 3621 & N/A & 0.00 & 515 & 0.97 & 37.4 \\
\hline NGC 2403 & N/A & 5.19 & 822 & 0.00 & 12.1 \\
\hline NGC 7793 & N/A & 0.00 & 286 & 2.82 & 18.8 \\
\hline NGC 2976 & N/A & 0.00 & 39.3 & 0.794 & 23.9 \\
\hline DDO 154 & N/A & 0.00 & 100 & 0.00 & 6.44 \\
\hline
\end{tabular}

Table 2. MOND fits for THINGS. MSE is in $(\mathrm{km} / \mathrm{s})^{2}$. Values in parentheses are from Gentile et al. fits minimizing reduced $\chi^{2}$ [59]. If there is no " $d$ constrained," the " $d$ free" fit satisfies " $d$ constrained." Corresponding plots are in Figures $3 \& 4$. *N/A means Gentile et al. did not use independent bulge data. ${ }^{* *}$ For comparison with Gentile et al. we fixed $\gamma_{\text {dist }}=1.09$. ${ }^{* * *}$ Gentile et al. used composite disk and bulge data in THINGS, while we used the separate disk and bulge data also supplied in THINGS.

\begin{tabular}{c|cccc} 
Name & $\gamma_{\text {bulge }}$ & $\gamma_{\text {disk }}$ & $\gamma_{\text {dist }}$ & MSE \\
\hline NGC 2841 & $0.99(1.02)$ & $0.79(0.70)$ & $1.46(1.53)$ & 19.5 \\
$d$ constrained & $1.09(1.24)$ & $1.32(1.20)$ & $1.11(1.11)$ & 54.1 \\
\hline NGC 7331 & $1.09(1.16)$ & $0.98(0.91)$ & $0.68(0.71)$ & 18.8 \\
$d$ constrained & $1.17(1.22)$ & $0.59(0.57)$ & $0.913(0.913)$ & 28.6 \\
\hline NGC 3521 & N/A & $1.77(1.08)$ & $0.44(0.65)$ & 210 \\
$d$ constrained & N/A & $0.93(0.97)$ & $0.70(0.70)$ & 264 \\
\hline NGC 6946 & $0.58(0.55)$ & $0.94(0.58)$ & $0.80(1.09)$ & 29.2 \\
comparison** & $0.49(0.55)$ & $0.59(0.58)$ & $1.09(1.09)$ & 32.3 \\
\hline NGC 2903 & $0.00(0.00)$ & $2.06(2.80)$ & $1.25(1.07)$ & 202 \\
\hline NGC 5055 & $0.40(0.47)$ & $0.80(0.84)$ & $0.66(0.62)$ & 26.7 \\
$d$ constrained & $0.38(0.43)$ & $0.72(0.70)$ & $0.70(0.70)$ & 27.2 \\
\hline NGC 3198*** & $0.37(\mathrm{~N} / \mathrm{A})$ & $1.59(\mathrm{~N} / \mathrm{A})$ & $0.58(0.62)$ & 18.8 \\
$d$ constrained & $0.26(\mathrm{~N} / \mathrm{A})$ & $0.65(\mathrm{~N} / \mathrm{A})$ & $0.89(0.89)$ & 74.2 \\
\hline NGC 3621 & N/A & $0.76(0.75)$ & $0.91(0.92)$ & 9.14 \\
\hline NGC 2403 & N/A & $0.69(0.63)$ & $1.53(1.46)$ & 34.4 \\
$d$ constrained & N/A & $1.29(1.10)$ & $1.08(1.08)$ & 42.3 \\
\hline NGC 7793 & N/A & $0.51(0.39)$ & $1.52(1.68)$ & 42.1 \\
$d$ constrained & N/A & $1.00(0.90)$ & $1.10(1.10)$ & 55.0 \\
\hline NGC 2976 & N/A & $0.11(0.09)$ & $2.02(2.17)$ & 7.83 \\
$d$ constrained & N/A & $0.38(0.36)$ & $1.10(1.10)$ & 12.0 \\
\hline DDO 154 & N/A & $4.01(2.47)$ & $0.64(0.71)$ & 1.63 \\
$d$ constrained & N/A & $1.36(1.56)$ & $0.75(0.75)$ & 4.60 \\
\hline & & & &
\end{tabular}




\section{X-ray Cluster Mass Profiles}

For fitting the mass profiles of X-ray clusters, one assumes a spherically symmetric, isothermal distribution of $\mathrm{X}$-ray emitting gas in hydrostatic equilibrium with the gravitational potential [17]. The gas mass profile as determined by the $\mathrm{X}$-ray luminosity is given by

$$
M_{p}(r)=4 \pi \int_{0}^{r} \rho\left(r^{\prime}\right) r^{\prime 2} d r^{\prime}
$$

where

$$
\rho(r)=\rho_{o}\left(1+\left(\frac{r}{r_{c}}\right)^{2}\right)^{-1.5 \beta} .
$$

The constants $\rho_{o}, r_{c}$ and $\beta$ are found from fits of the surface density map $\Sigma(x, y) . M_{p}(r)$ corresponds to the "proper mass" for galactic RC's above. The dynamical mass profile needed for hydrostatic equilibrium at temperature $T$ is given by

$$
M(r)=\frac{3 \beta k T}{G \mu_{A} m_{p}}\left(\frac{r^{3}}{r^{2}+r_{c}^{2}}\right)
$$

where $\mu_{A}$ is the mean atomic weight of the gas constituents $(\approx 0.609), m_{p}$ is the mass of the proton, $k$ is the Boltzmann constant, and $G$ is the gravitational constant. In the MSTG version of MOG per Moffat \& Brownstein, as stated above, $G \rightarrow G(r)$ and is understood to reduce $M(r)$ of Eq(19) to $M_{p}(r)$ of Eq(17). Effectively, we have

$$
M_{p}(r)=M(r)+M_{o} \eta(r)-\sqrt{\left(M_{o} \eta(r)\right)^{2}+2 M_{o} M(r) \eta(r)}
$$

where

$$
\eta(r)=0.5\left(1-\exp \left(-r / r_{o}\right)\left(1+\frac{r}{r_{o}}\right)\right)^{2}
$$

with $M_{o}$ and $r_{o}$ fitting parameters and $M(r)$ computed using $G$ in $\mathrm{Eq}(19)$.

Since we're assuming spherical symmetry, our approach used for galactic RC's can be easily adopted here. Contrary to MSTG, we are increasing $d M_{p}(r)$ by a geometric factor to give the dynamical mass $M(r)$. Thus, we modify $\mathrm{Eq}(17)$ to give

$$
M(r)=\int_{0}^{r} \sqrt{B+\frac{A}{r^{\prime}}} d M_{p}=4 \pi \int_{0}^{r} \sqrt{B+\frac{A}{r^{\prime}}} \rho\left(r^{\prime}\right) r^{\prime 2} d r^{\prime}
$$

with $\rho(r)$ given by Eq(18) and $A$ and $B$ fitting parameters in analogy to our fits of galactic RC's. This value of $M(r)$ is then compared to that of $\mathrm{Eq}(19)$.

Here we will compare our ansatz to MSTG for fitting the mass profiles of the eleven X-ray clusters found in Brownstein [57] as obtained from Reiprich and Böhringer [62,63] using combined ROSAT and ASCA data. All data were taken from Brownstein, as well as the parameters for the MSTG best fits, since therein Brownstein found MSTG fits of these X-ray clusters bested those of MOND and STVG. As with our comparison of our ansatz to MOND for galactic RC's, one should keep in mind that MSTG, as a well-developed theory, is more constrained than our ansatz, so the comparison between our ansatz and MSTG here is only for establishing the feasibility of our approach. In each case, we computed proper (or "gas") mass $M_{p}(r)$ and dynamical mass $M(r)$ at eleven logrithmically evenly spaced points from $13 \mathrm{kpc}$ to $r_{\text {out }}$, "the point where the density of the X-ray cluster drops to $\approx 10^{-28} \mathrm{~g} / \mathrm{cm}^{3}$ which is about 250 times the mean cosmological density of baryons." The values of $T, \rho_{0}, \beta$, and $r_{c}$, supplied by Brownstein, were used with Eqs(17-19) to compute $M_{p}(r)$ and $M(r)$. We then computed the MSTG best fit values for $M_{p}(r)$ at these eleven locations using $M_{o}$ and $r_{o}$, supplied by Brownstein, with Eqs(20 \& 21). Our best values of $A$ and $B$ were found by fitting the dynamical 
Table 3. Our fits for mass profiles of $\mathrm{X}$-ray clusters. MSE is $(\Delta \log (M))^{2}$. Corresponding plots are in Figures $5 \& 6$.

\begin{tabular}{c|ccc} 
Name & $A$ & $B$ & MSE \\
\hline Bullet & 826 & 87.5 & 0.00221 \\
Abell 2142 & 21500 & 34.0 & 0.00786 \\
Coma & 10500 & 198 & 0.0105 \\
Abell 2255 & 1430 & 202 & 0.00256 \\
Perseus & 27800 & 0.00 & 0.00173 \\
Norma & 30300 & 105 & 0.0159 \\
Hydra-A & 30600 & 15.5 & 0.00081 \\
Centaurus & 130000 & 0.00 & 0.00737 \\
Abell 400 & 47000 & 27.2 & 0.00848 \\
Fornax & 20900 & 2380 & 0.00126 \\
Messier 49 & 999000 & 3440 & 0.00018
\end{tabular}

Table 4. MSTG fits for mass profiles of X-ray clusters. $M_{o}$ and $r_{o}$ were taken from Brownstein [57]. MSE is $(\Delta \log (M))^{2}$. Corresponding plots are in Figures $5 \& 6$.

\begin{tabular}{c|ccc} 
Name & $\begin{array}{c}M_{o} \\
\left(10^{14} M_{\odot}\right)\end{array}$ & $\begin{array}{c}r_{o} \\
(\mathrm{kpc})\end{array}$ & MSE \\
\hline Bullet & 56.7 & 116.8 & 0.0443 \\
Abell 2142 & 30.0 & 56.8 & 0.0302 \\
Coma & 30.7 & 88.2 & 0.0342 \\
Abell 2255 & 43.8 & 157.4 & 0.0386 \\
Perseus & 10.7 & 23.5 & 0.0239 \\
Norma & 30.1 & 97.6 & 0.0373 \\
Hydra-A & 9.5 & 23.9 & 0.0348 \\
Centaurus & 10.0 & 14.2 & 0.0218 \\
Abell 400 & 6.0 & 44.7 & 0.0124 \\
Fornax & 13.7 & 67.4 & 0.0318 \\
Messier 49 & 10.3 & 10.8 & 0.0244
\end{tabular}

mass $M(r)$ of $\mathrm{Eq}(22)$. As with galactic RC's, we computed a simple MSE to quantify the fits (MSE is logarithmic, since we did a log-log plot of the fits). The plots of these best fits are in Figures $5 \& 6$. Our results are in Table 3 and MSTG results are in Table 4.

We also point out that gravitational lensing data of the Bullet Cluster (1E0657-558) originally touted as "direct empirical proof of the existence of dark matter," [64] can be explained without DM $[17,23,65]$. What happened in this case is a small galactic cluster ("subcluster") collided with the larger Bullet Cluster. The galaxies of both clusters passed through the collision region relatively unaffected, but the intracluster medium (ICM) gas of the two clusters was left behind in the collision region. The result was four lobes of baryonic matter aligned as follows: the galaxies of the Bullet Cluster, the gas of the Bullet Cluster, the gas of the subcluster, and the galaxies of the subcluster (Figure 1). If one accepts that the mass of the cluster galaxies is only $10 \%$ of the baryonic mass, then in the absence of DM one would expect gravitational lensing maps of this region (blue lobes in Figure 1) to overlap X-ray images of the gas lobes (red lobes in Figure 1), since the gas possesses $90 \%$ of the baryonic mass. What Clowe et al. rather found [64] was that the lensing peaks were located in the galaxy lobes, so the galaxies are inside the blue lobes of Figure 1. Their conclusion was that there exists large quantities of DM which passed through the collision with the galaxies. Brownstein \& Moffat $[17,65]$ and Israel \& Moffat [23] explained the offset lensing peaks using MOG because $G(r)$ associated with the galaxies increases more than $G(r)$ associated with the gas, since the galaxies are farther removed from the center of the Newtonian gravitational potential. The explanation in terms of our ansatz would be 


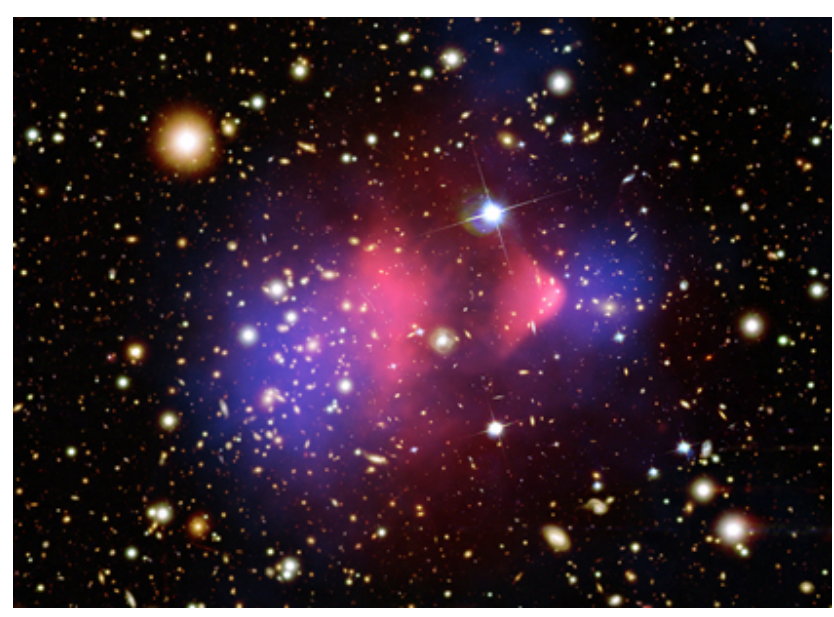

Figure 1. Bullet Cluster X-ray and lensing composite (false color) image from NASA Release 06-297. Blue lobes are lensing data in the vicinity of the galaxies. Red lobes are X-ray images of ICM gas left behind after the subcluster (right side) passed through the Bullet Cluster (left side).

similar, since $G$ is associated with $\rho$ in Einstein's equations, i.e., the proper mass of each of the galaxy and gas lobes is increased to a larger dynamical mass. Since the galaxies are farther removed from the center of the Newtonian gravitational potential, the dynamical mass of the galaxies exceeds the dynamical mass of the gas leading to lensing peaks in the galaxy lobes. A fit of the offset using our ansatz at this point is of no interest, since the number of fitting parameters would equal the number of data points. Thus, fitting these lensing data will have to wait until the fitting parameters of our ansatz are motivated physically.

\section{CMB Angular Power Spectrum}

After the Bullet Cluster, the most compelling evidence for DM is perhaps the CMB angular power spectrum [66]. In this situation, small primordial inhomogeneities $\Delta_{i}=\frac{\delta \rho_{i}}{\rho_{i}}$ in the otherwise homogeneous energy density $\rho$ of the the background FLRW cosmology model lead to anisotropies in the CMB angular power spectrum $C_{\ell}$ versus $\ell$ (typically plotted $D_{\ell}$ versus $\ell$ where $D_{\ell}=\frac{\ell(\ell+1) C_{\ell}}{2 \pi}$ ). The various components $\Delta_{i}$ typically considered are photons, baryons (this term includes electrons), neutrinos, and DM. Before recombination, the photons are coupled to the baryons and together their perturbations oscillate, gravity pulling them in while the photon gas pressure pushes them out. The neutrino perturbations simply stream free and the DM perturbations stay put, since DM is not affected by the photon gas pressure. As a result, the DM acts to enhance the contraction phase of the photon-baryon $\Delta_{i}$ oscillations while suppressing the rarefaction phase which manifests itself as enhanced odd peaks relative to even peaks in $D_{\ell}$ versus $\ell$. Thus, the size of the third peak relative to the second peak is taken to be an indication of DM and the Planck 2015 CMB data clearly show that the second and third peaks have very nearly the same amplitude (Figure 2) in accord with the existence of DM. Therefore, it is this effect that we wish to reproduce without DM using our ansatz and STVG.

The relativistic version of MOND known as the Tensor-Vector-Scalar (TeVeS) theory, does not show such an enhanced third peak [67,68], so the Planck 2015 CMB data would appear to present a serious challenge for MOND [69], although Skordis is working on a new approach and expects results in 2017 [70]. Angus [71] has argued that non-relativistic MOND would have no cosmological effect and one can obtain the enhanced third peak using a sterile neutrino with mass $11 \mathrm{eV}$. This also allows MOND to account for "the dark matter of galaxy clusters without influencing individual galaxies, ... and potentially fit the matter power spectrum." Whether this constitutes a true extension of MOND 
or a "cheat" is subject to debate, as cosmology certainly resides in the relativistic domain, so some relativistic version of MOND that accounts for the CMB anisotropies is needed [72].

The situation with MOG is less contentious, as its practitioners seem to agree that it has addressed astronomical data at all scales with some degree of success. Recently, Moffat \& Toth [73] used STVG to successfully account for the matter power spectrum per data from the Sloan Digital Sky Survey, as well as the CMB angular power spectrum per data from WMAP and the Boomerang experiment for $\ell \leq 1000$, which includes the third peak. In both cases, they used an enhanced gravitational constant $G$ in a fashion similar to the MOG account of the Bullet Cluster. Specifically, Moffat \& Toth [73] modified $G$ in Mukhanov's analytic approach [74] such that, "When a quantity containing $G$ appears in an equation describing a gravitational interaction, $G_{e f f}$ must be used. However, when a quantity like $\Omega_{b}$ is used to describe a nongravitational effect, the Newtonian value of $G$ must be retained." The modification is given by

$$
G_{e f f}=G\left[1+\alpha\left[1-\left(1+\frac{\mu a}{k}\right) \exp \left(\frac{\mu a}{k}\right)\right]\right]
$$

$\Omega_{o}$ is the current total energy density divided by the critical energy density (required to make flat spatial hypersurfaces in FLRW cosmology). $\Omega_{m}$ is the current contribution to the total energy density due to matter. In the model herein, we have $\Omega_{m}=\Omega_{0}$, i.e., $\Omega_{\Lambda}=0$ and the current photon and neutrino contributions are negligible. $\Omega_{b}$ is the current contribution to the total energy density due to baryons, so for our ansatz and STVG, $\Omega_{b}=\Omega_{m}$, i.e., $\Omega_{D M}=0$. Since STVG is the best modified gravity fit of the $\mathrm{CMB}$ angular power spectrum to date, we will compare our fitting procedure to that of STVG. As it turns out, both can reproduce the standard cold dark matter (sCDM) fit of the first three acoustic peaks in a rather trivial fashion. To show this, we need to look carefully at an sCDM fitting procedure. Before we start, we point out that STVG gets rid of DM but it retains $\Lambda$ while we get rid of both DM and $\Lambda$. Therefore, the comparison of STVG and our ansatz in this context will only involve the replacement of DM in SCDM.

The analytic method of Hu \& Sugiyama $[75,76](\mathrm{HuS})$ maps closely to the full numerical solution in the first three acoustic peaks and its physics is transparent, so we will use HuS. Since the Planck 2015 data strongly suggest a spatially flat universe [77], i.e., $\Omega_{0}=1$, we will work in that context. Further, we have no $\Lambda$, so $\Omega_{m}=\Omega_{o}=1$ while a proper STVG fit would have $\Omega_{m} \approx 0.3$ and $\Omega_{\Lambda} \approx 0.7$. Here is a brief outline of HuS in this context with an explanation of our ansatz and STVG modifications thereto.

The spatially flat FLRW metric (we will use c $=1$ in what follows) [55]

$$
d s^{2}=-d t^{2}+a^{2}(t) \delta_{i j} d x^{i} d x^{j}
$$

is modified in the conformal Newtonian gauge by the existence of the perturbations to read

$$
d s^{2}=-(1+2 \Psi) d t^{2}+(1+2 \Phi) a^{2}(t) \delta_{i j} d x^{i} d x^{j}
$$

where $\Psi$ is called "the Newtonian potential" and $\Phi$ is a perturbation to the spatial curvature. When pressure is negligible, $\Psi=-\Phi$. Since the spatial part of the Schwarzschild metric can be written [58]

$$
d s^{2}=\left(1+\frac{G M}{2 r}\right)^{4} \delta_{i j} d x^{i} d x^{j}
$$

we have for small $\frac{G M}{2 r}\left(=-\frac{\Phi}{2}\right)$

$$
d s^{2} \approx\left(1+\frac{2 G M}{r}\right) \delta_{i j} d x^{i} d x^{j}
$$


which explains the perturbation $\Phi$ in $\mathrm{Eq}(25)$ as a small Newtonian potential placed in a spatially flat FLRW background. Thus, as with galactic rotation curves and X-ray cluster mass profiles, we will be correcting what is already a small effect, i.e., $\Psi$ and $\Phi$. STVG will be correcting $G$ as shown above. Einstein's equations directly relate $\Psi$ and $\Phi$ to the total energy density $\rho$, which only appears coupled to $G$. That is, Einstein's equations give

$$
k^{2} \Phi=4 \pi G \rho\left(\frac{a}{a_{0}}\right)^{2} \Delta_{T}
$$

and

$$
\Phi+\Psi=-\frac{8 \pi G \rho}{k^{2}}\left(\frac{a}{a_{0}}\right)^{2} \Pi
$$

in the total matter rest frame gauge in $k$ space where $\Pi$ is the anisotropic stress and $\Delta_{T}$ is the total perturbation. Thus, a multiplicative correction to $\Psi$ and $\Phi$ on the LHS of Eqs(28 \& 29) per our ansatz could equally be viewed as a multiplicative STVG correction to $G$ on the RHS. Eqs(28 \& 29) are represented in HuS as

$$
\bar{\Phi}(a, k)=\frac{9}{10}\left(\frac{k_{e q}}{k}\right)^{3 / 2} \frac{a+1}{a^{2}}\left[1+\frac{2}{5} f_{v}\left(1-\frac{1}{3} \frac{a}{a+1}\right)\right] U_{G}(a)
$$

and

$$
\bar{\Psi}(a, k)=-\frac{6}{5}\left(\frac{k_{e q}}{k}\right)^{2} \frac{a+1}{a^{2}} f_{v} \frac{\bar{N}_{2}}{(a+1)}-\bar{\Phi}(a, k)
$$

where $f_{v}$ is the fraction of the radiation energy density contributed by the neutrinos (0.405),

$$
U_{G}(a)=\frac{9 a^{3}+2 a^{2}-8 a-16+16 \sqrt{a+1}}{9 a(a+1)}
$$

and

$$
\bar{N}_{2}=\frac{12}{45 a} \frac{\sqrt{k}}{\sqrt{\mathrm{keq}}}\left[8+4 a-3 a^{2}-8 \sqrt{1+a}+4 a \log \frac{a}{4}+4 a \log \left(\frac{\sqrt{1+a}+1}{\sqrt{1+a}-1}\right)\right]
$$

is the neutrino quadrupole contribution to the anisotropic stess with

$$
k_{e q}=7.46 \times 10^{-2}\left(\frac{\mathrm{T}_{0}}{2.7}\right)^{-2} h^{2} \Omega_{0}
$$

$T_{o}$ is the current temperature of the CMB and $h$ is the current Hubble constant divided by 100 $\mathrm{km} / \mathrm{s} /$ Mpc. Again, Planck data strongly suggest $\Omega_{0}=1$, so we will use that model. The overbar represents values prior to contributions from the matter transfer function $T(k)$. To account for that

$$
\Phi(a, k)=\bar{\Phi}\left((1-T(k)) e^{-\alpha_{1}\left(\frac{a k}{k_{e q}}\right)^{\beta}}+T(k)\right)
$$

and

$$
\Psi(a, k)=\bar{\Psi}\left((1-T(k)) e^{-\alpha_{2}\left(\frac{a k}{k_{e q}}\right)^{\beta}}+T(k)\right)
$$

where

$$
T(k)=\frac{\log (1+2.34 q)}{2.34 q}\left[(6.71 q)^{4}+(5.46 q)^{3}+(14.1 q)^{2}+3.89 q+1\right]^{-1 / 4}
$$


with $q=k e^{2 \Omega_{b}} /\left(h^{2} \Omega_{m}\right) . \alpha_{1}, \alpha_{2}$, and $\beta$ are fitting factors which Hu \& Sugiyama found to be $0.11,0.097$ and 1.6, respectively. Now we can find the monopole and dipole CMB temperature perturbations at recombination $\left(\Theta_{0}\left(\eta_{\star}\right)\right.$ and $\Theta_{1}\left(\eta_{\star}\right)$, respectively) before damping by solving

$$
\Theta_{0}(\eta)=-\frac{k}{3} \Theta_{1}(\eta)-\dot{\Phi}(\eta)
$$

and

$$
\Theta_{1}(\eta)=-\frac{\dot{R}(\eta)}{1+R(\eta)} \Theta_{1}(\eta)+\frac{1}{1+R(\eta)} k \Theta_{0}(\eta)+k \Psi(\eta)
$$

for $k>0.08 h^{3}$ and the same equations with $R(\eta)=0$ for $k \leq 0.08 h^{3}$ from $\eta=0$ to $\eta=\eta_{\star}$, which is the conformal time at recombination. [The derivatives are with respect to conformal time $\eta$.] We have

$$
a(\eta)=\frac{1}{8}\left(4 \sqrt{2} k_{e q} \eta+k_{e q}^{2} \eta^{2}\right)
$$

and

$$
R(\eta)=\frac{1}{1-f_{v}} \frac{3 \Omega_{b}}{4 \Omega_{0}} a(\eta)
$$

where $a(\eta)$ is normalized at matter-radiation equality $a_{e q}=1$. We also have

$$
a\left(\eta_{0}\right)=\frac{\Omega_{o} h^{2}}{4 \times 10^{-5}\left(\frac{T_{0}}{2.7}\right)^{4}}
$$

and the redshift at recombination

$$
z_{\star}=1000 \Omega_{b}^{-c 1 / c 2}
$$

with $c 1=0.43$ and $c 2=16+1.8 \log \left(\Omega_{b}\right)$. The $\mathrm{CMB}$ temperature perturbations are then damped due to diffusion during recombination

$$
\begin{gathered}
\Theta_{0}\left(\eta_{\star}\right)+\Psi\left(\eta_{\star}\right) \rightarrow e^{-\left(\frac{k}{k_{D}}\right)^{m}}\left(\Theta_{0}\left(\eta_{\star}\right)+\Psi\left(\eta_{\star}\right)\right) \\
\Theta_{1}\left(\eta_{\star}\right) \rightarrow e^{-\left(\frac{k}{k_{D}}\right)^{m}} \Theta_{1}\left(\eta_{\star}\right)
\end{gathered}
$$

where

$$
\begin{gathered}
m=1.46\left(h^{2} \Omega_{o}\right)^{0.0303}\left(1-0.128 \tan ^{-1}\left[\log \left(32.8 h^{2} \Omega_{b}\right)^{0.643}\right]\right) \\
k_{D}=F_{1}\left(\frac{2}{\pi} \tan ^{-1}\left[\frac{\pi}{2}\left(h^{2} \Omega_{b}\right)^{p_{2}}\left(\frac{F_{2}}{F_{1}}\right)^{p_{2} / p_{1}}\right]\right)^{p_{1} / p_{2}} \\
p_{1}=0.25, \quad p_{2}=2.38\left(h^{2} \Omega_{o}\right)^{0.184} \\
F_{1}=0.293\left(h^{2} \Omega_{o}\right)^{0.545}\left(\frac{1}{\left(25.1 h^{2} \Omega_{o}\right)^{0.648}}+1\right)
\end{gathered}
$$

and

$$
F_{2}=0.524\left(h^{2} \Omega_{0}\right)^{0.505}\left(\frac{1}{\left(10.5 h^{2} \Omega_{o}\right)^{0.564}}+1\right)
$$


Next the damped monopole and dipole perturbations at $\eta_{\star}$ are free streamed to higher $\ell$ today

$$
\begin{aligned}
\Theta_{\ell}\left(\eta_{o}\right)=\left[\Theta_{o}\left(\eta_{\star}\right)+\Psi\left(\eta_{\star}\right)\right](2 \ell+1) j_{\ell}\left(k \Delta \eta_{\star}\right)+ \\
\Theta_{1}\left(\eta_{\star}\right)\left[\ell j_{\ell-1}\left(k \Delta \eta_{\star}\right)-(\ell+1) j_{\ell+1}\left(k \Delta \eta_{\star}\right)\right]+(2 \ell+1) \text { ISW }
\end{aligned}
$$

where $j_{\ell}(z)$ is the spherical Bessel function, $\Delta \eta_{\star}=\eta_{0}-\eta_{\star}$, and the integrated Sachs-Wolfe effect is

$$
\mathrm{ISW}=\int_{\eta_{\star}}^{\eta_{0}}[\dot{\Psi}-\dot{\Phi}] j_{\ell}(k \Delta \eta) d \eta
$$

with $\Delta \eta=\eta_{o}-\eta$. We used the HuS approximation

$$
\mathrm{ISW} \approx[\Psi-\Phi]_{\eta_{\star}}^{\eta_{o}} j_{\ell}\left(k \Delta \eta_{\star}\right)
$$

Finally, the $\Theta_{\ell}\left(\eta_{0}, k\right)$ are integrated to give

$$
\frac{2 \ell+1}{4 \pi} C_{\ell}=\frac{V}{2 \pi^{2}} \int \frac{d k}{k} k^{3} \frac{\left|\Theta_{\ell}\left(\eta_{o}, k\right)\right|^{2}}{2 \ell+1}
$$

where $V$ is an overall normalization constant. We used 1000 values of $k$ in the interval $\frac{\pi}{\eta_{o}} \leq k \leq k_{D}$ for each of the 46 points in the HuS fit shown in Figure 2. Primarily, one has only $\Omega_{b}$ and $h$ for this HuS sCDM fit, but we adjusted a few others, viz., $m$ in the damping factor $(1.46 \rightarrow 1.10(5))$, the equation for redshift at decoupling $(1000 \rightarrow 1080(5))$, and $p 1$ in the equation for the damping factor $(0.25 \rightarrow 0.23(1))$, to obtain our HuS fit. While $h$ is certainly smaller than accepted $(0.49(1)$ instead of $\sim 0.7$ ) and $\Omega_{b}$ is too large (0.090(1) instead of $\left.\sim 0.05\right)$, the combination $h^{2} \Omega_{b}$ is close to the accepted range $(0.02222 \pm 0.00023)$ [77]. Again, the point of the fit isn't to find the fit parameters per se, although that will certainly be important once we are at the stage of building a cosmology model. Rather, we want to find out what change is required to bring the third peak up to the second peak without DM in the context of our ansatz and compare that with an established program in modified gravity, STVG.

In order to remove the DM from this $\mathrm{SCDM}$ fit, we look to the very first step Eqs(30 \& 31) since that is the representation of Einstein's equations in the metric perturbations $\Psi$ and $\Phi$. Therein, we see that the only place matter of any kind enters the equations is through $k_{e q}$ by way of $h^{2} \Omega_{m}$. Of course, that's because $h^{2} \Omega_{m} \propto G \rho_{m}\left(a_{0}\right)$. Thus, eliminating DM from $\rho_{m}\left(a_{0}\right)$ means a loss of $91 \%$ of the critical density necessary for $\Omega_{m}=\Omega_{0}=1$ (in this particular HuS fit). The correction needed to restore the HuS fit, having removed the DM, is straightforward and exact. Since we have $\Omega_{m} \rightarrow \Omega_{b}$ in removing $\mathrm{DM}$, in order to restore the HuS fit, we must multiply $h^{2} \Omega_{m}$ by $\Omega_{b}^{-1}$, i.e., $\Omega_{b}^{-1}\left(h^{2} \Omega_{m} \rightarrow h^{2} \Omega_{b}\right)=h^{2}=$ $h^{2} \Omega_{m}$. In order to have that change to $k_{e q}$ in our view, $\Phi$ and $\Psi$ are multiplied by $\Omega_{b}^{-3 / 2}$, since they are both proportional to $k_{\text {eq }}^{3 / 2}$. In STVG, we simply need $G \rightarrow \Omega_{b}^{-1} G$ which Eq(23) gives for $\mu=0$ and $\alpha=\Omega_{b}^{-1}-1^{2}$.

While our ansatz is couched in real space and this correction is in $k$ space, the correction factor is a constant, so it is the same in both spaces. Thus, our ansatz correction to $\Phi$ and $\Psi$ is simply $A=0$ and $B=\Omega_{b}^{-3}$. As with the corrected Newtonian potentials above, even though this correction is much larger than 1 , the corrected $\Phi$ and $\Psi$ are still much less than 1 (they were already small including DM). Thus, this particular fit says that the mass of the baryons in the context of Big Bang nucleosynthesis is only approximately $10 \%$ of that needed for critical density $\Omega_{m}=1$, so if the early universe had not contained the small bound baryon-photon perturbations, $\Omega_{m}$ would have been $\sim 0.1$ and the perfectly smooth universe would have evolved per the open FLRW model. However, the

2 The $k$ dependence is necessary for the matter power spectrum, so nonzero $\mu$ is required there. 


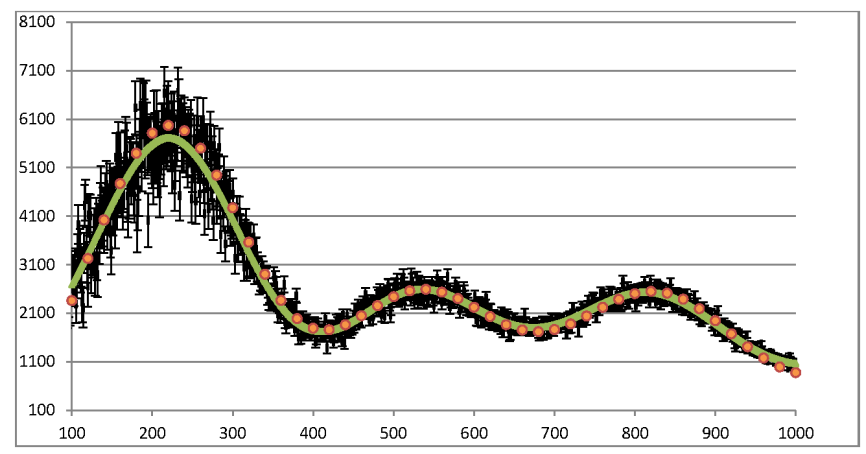

Figure 2. This is a plot of $D_{\ell}$ in $(\mu K)^{2}$ versus $\ell$ in the range $100 \leq \ell \leq 1000$ for the Planck 2015 CMB data [78] (black error bars), the Planck consortium's best $\Lambda$ CDM fit [79] (solid line), and our best HuS sCDM fit (dots). Our ansatz and STVG trivially reproduce the HuS fit without DM. Since we do not have $\Lambda$, our best fit to these data would equal the HuS sCDM best fit. The root-mean-square error (RMSE) for the HuS sCDM fit points shown is $225(\mu \mathrm{K})^{2}$. STVG can also trivially replace DM in $\Lambda \mathrm{CDM}$ and STVG keeps $\Lambda$, so the STVG best fit to these data would equal the $\Lambda$ CDM best fit. The RMSE for the $\Lambda$ CDM fit shown corresponding to the HuS fit points shown is $240(\mu \mathrm{K})^{2}$, although this fit is for all $\ell$ in the range $30 \leq \ell \leq 2508$.

bound baryon-photon perturbations enhanced the mass of the smooth background matter contained therein rendering $\Omega_{m}=1$, so the universe evolved per the spatially flat FLRW model. Of course, the baryon-photon perturbations seeded the large scale structure we observe today, so they are necessary to explain that as well.

Again, in STVG, wherever you have $\Omega_{m} h^{2}$ (i.e., you have $G \rho_{m}\left(a_{0}\right)$ ) in your fitting procedure, $G \rightarrow \Omega_{b}^{-1} G$ exactly restores the loss of $\Omega_{D M}$ from $\Omega_{m}$. In the standard best fit for Planck 2015, i.e., $\Lambda \mathrm{CDM}, \Omega_{m} \approx 0.3$ and $\Omega_{b} \approx 0.05$, so $G \rightarrow 6 G$ allows baryons alone to do the work of DM plus baryons in the $\Lambda \mathrm{CDM}$ fit. That is what Moffat \& Toth found [73] using $\mu^{-1} \approx$ particle horizon, which is $\mu \approx 0$, so $G \rightarrow(1+\alpha) G$. Since $G \rightarrow G_{\text {eff }}$ per STVG regardless of the bound baryon-photon perturbations, the primordial perturbations aren't necessary to produce the apparent enhanced mass of the baryons.

\section{Conclusion}

As already provided by GR, baryonic matter on astronomical scales can have have two values of mass that we called "proper mass" and "dynamical mass." We used this fact to argue that the dynamical mass of the rarefied, gravitationally bound baryonic matter in galaxies, galactic clusters, and primordial density perturbations is currently attributed to baryonic matter plus non-baryonic dark matter (DM). While the dynamical mass of baryonic matter can be many times larger than its proper mass, this mass difference can be accounted for by a small difference in the spacetime geometry. That is, small geometric differences in spacetime structure on astronomical scales can generate large proper and dynamical mass differences, which is how one can understand MOND as well.

We previously used an ansatz based on GR perturbation theory for fitting the SCP Union2.1 SN Ia supernova data without dark energy. Since the ansatz was successful in fitting those data without dark energy, we generalized it here for computing dynamical mass from proper mass in fitting THINGS galactic rotation curve data, ROSAT / ASCA X-ray cluster mass profile data, and Planck 2015 $\mathrm{CMB}$ angular power spectrum data without DM. We compared our ansatz fits in each case with those of more advanced modified gravity programs, i.e., MOND, MSTG, and STVG, respectively, and found that our fits compared favorably in all three cases. While this compatibility of fits does not establish the validity of our proposition, it does provide confidence in using the empirically derived 
values of the constants in our ansatz to pursue an underlying action. For example, we will explore the possibility that the arbitrary constants in our ansatz have a basis in astrometric quantities, since the results show interesting trends in the fitting factors $A$ and $B$ of our metric correction. That is, in Table 1 for our galactic RC fits we see no correction in the six bulge contributions $A_{b u l g e}$ and only a relatively small correction in five of twelve disk contributions $A_{\text {disk, }}$, while there were relatively large corrections to all twelve gas contributions $A_{g a s}$. The constant $B$ was small to nonexistent in all twelve cases. Moving to Table 3 for our X-ray cluster mass profile fits we see again large corrections to the ICM gas mass $A$ with an increased contribution from the constant $B$. For the primordial density perturbations, $A=0$ and the correction is entirely in $B$. Therefore, it would appear that the functional form for our ansatz is relevant and it's not unreasonable to search for astrometric hints of an underlying action in these fits.

No matter how we do it, we must ultimately produce a physical basis for the ansatz fitting factors of the SCP Union2.1 SN Ia, THINGS, ROSAT/ASCA, and Planck 2015 data. Ideally, this will be in the form of an underlying action, but it could be empirical [51]. Given the physical motivation for the otherwise arbitrary constants of our ansatz, it will be possible to construct a commensurate cosmology model that will then account coherently for data on all astrophysical scales, to include the matter power spectrum. That is the ultimate goal of our program.

Acknowledgments: We thank Erwin de Blok for providing the THINGS data, Gianfranco Gentile for providing information about his MOND fits, Joel Brownstein for providing information about MSTG, Thomas Reiprich for providing information about X-ray cluster mass data, Stacy McGaugh for providing input about MOND as relates to cosmology, Wayne Hu for help with HuS, and Viktor Toth for help with the STVG fit of Planck 2015.

Conflicts of Interest: The authors declare no conflict of interest.

\section{References}

1. Oort, J.H.: The force exerted by the stellar system in the direction perpendicular to the galactic plane and some related problems. Bulletin of the Astronomical Institutes of the Netherlands 6, 249-287 (1932).

2. Zwicky, F.: Spectral displacement of extra galactic nebulae. Helvetica Physica Acta 6, 110-127 (1933).

3. Zwicky, F.: On the masses of nebulae and clusters of nebulae. The Astrophysical Journal 86, 217-246 (1937).

4. Rubin, V., and Ford, K: Rotation of the Andromeda nebula from a spectroscopic survey of emission regions. The Astrophysical Journal 159, 379-403 (1970).

5. Garrett, K., and Duda, G.: Dark Matter: A Primer. Advances in Astronomy 2011, doi:10.1155/2011/968283 (2011).

6. Munoz, C.: Dark matter detection in the light of recent experimental results. International Journal of Modern Physics A 19, 3093-3170 (2004).

7. Feng, J.: Dark Matter Candidates from Particle Physics and Methods of Detection. Annual Reviews of Astronomy and Astrophysics 48, 495 (2010) http:/ / arxiv.org/abs/1003.0904

8. Milgrom, M.: A modification of the Newtonian dynamics as a possible alternative to the hidden mass hypothesis. The Astrophysical Journal 270, 365-370 (1983).

9. Milgrom, M: MOND theory. Canadian Journal of Physics 93, 107-118 (2015) http://arxiv.org/abs/1404.7661

10. Sanders, R.H., and McGaugh, S.: Modified Newtonian Dynamics as an Alternative to Dark Matter. Annual Reviews of Astronomy \& Astrophysics 40, 263-317 (2002) http:/ /arxiv.org/abs/astro-ph/0204521

11. Bekenstein, J.D.: Relativistic gravitation theory for the modified Newtonian dynamics paradigm. Physical Review D 70, 083509 (2004).

12. Sanders, R.H.: A tensor-vector-scalar framework for modified dynamics and cosmic dark matter. Monthly Notices of the Royal Astronomical Society 363, 459-468 (2005).

13. Zlosnik, T., Ferreira, P., and Starkman, G.: Modifying gravity with the aether: An alternative to dark matter. Physical Review D 75, 044017 (2007) http:/ /arxiv.org/abs/astro-ph/0607411

14. Zhao, H.S., and Li, B.: Dark Fluid: A Unified framework for Modified Newtonian Dynamics, Dark Matter, and Dark Engery. The Astrophysical Journal 712, doi:10.1088/0004-637X/712/1/130 (2010). 
NGC 2841

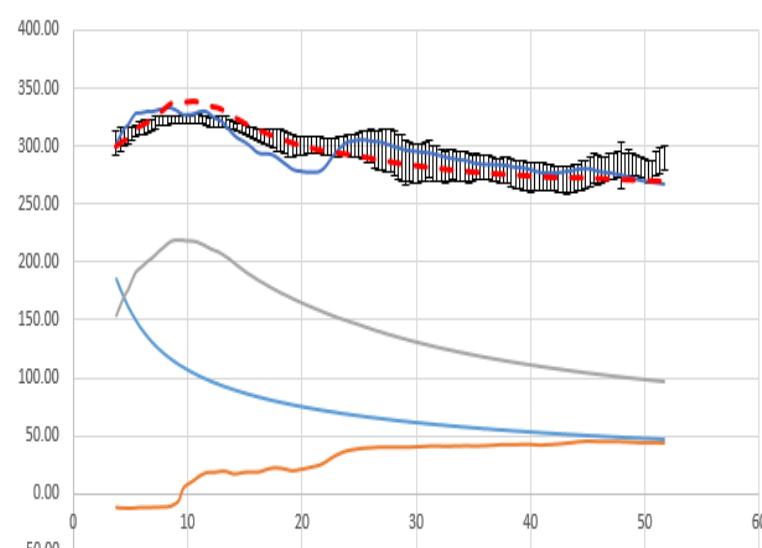

NGC 3521
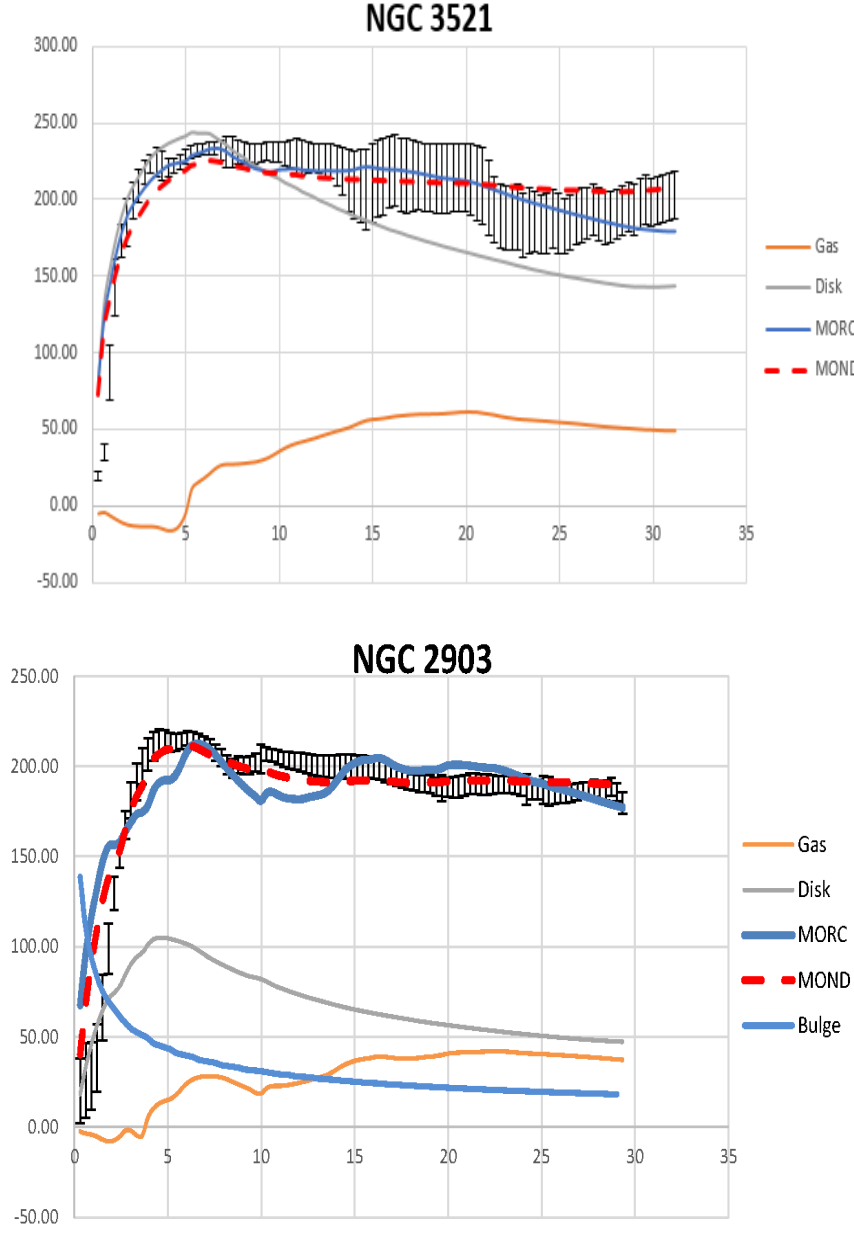

NGC 7331

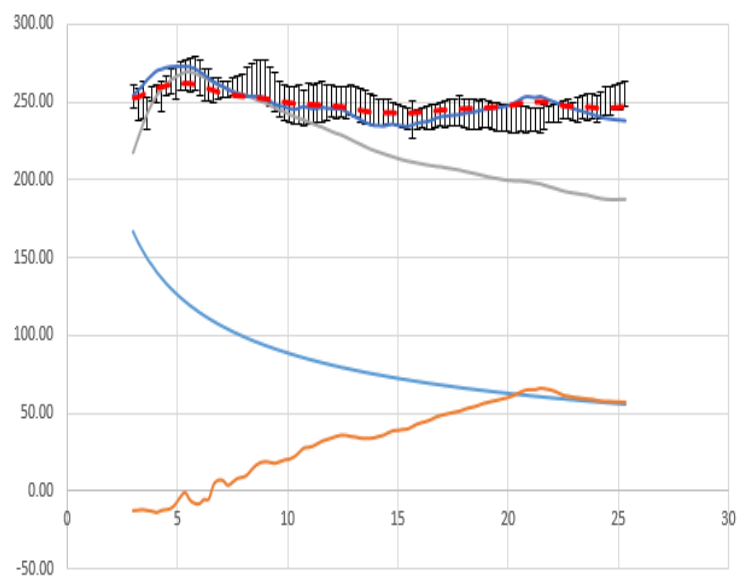

NGC 6946

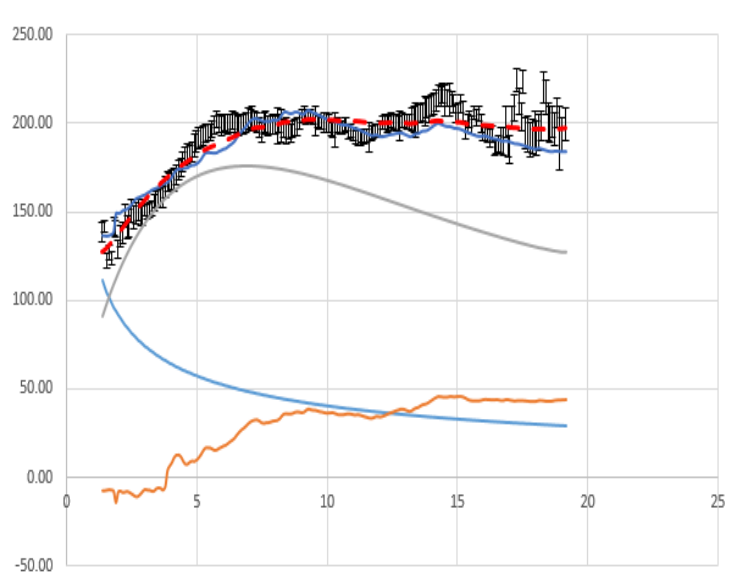

NGC 5055

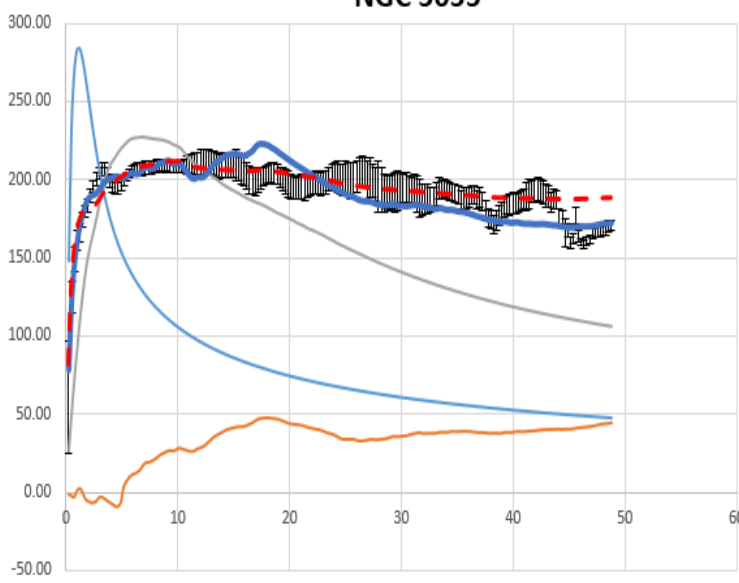

Figure 3. Graphs of our fits (solid) and MOND fits (dashed) of THINGS galactic RC's (with error bars). Our fit disappears when it lies right underneath MOND. Disk, gas and bulge curves are labeled. Bulge curves are not always available. Vertical axis is rotation velocity in $\mathrm{km} / \mathrm{s}$ and horizontal axis is orbital radius in kpc. Note: The MOND distance fitting factor $\gamma_{\text {dist }}$ would alter the horizontal scale proportionally. Corresponding numerical results are in Tables $1 \& 2$. 
NGC 3198

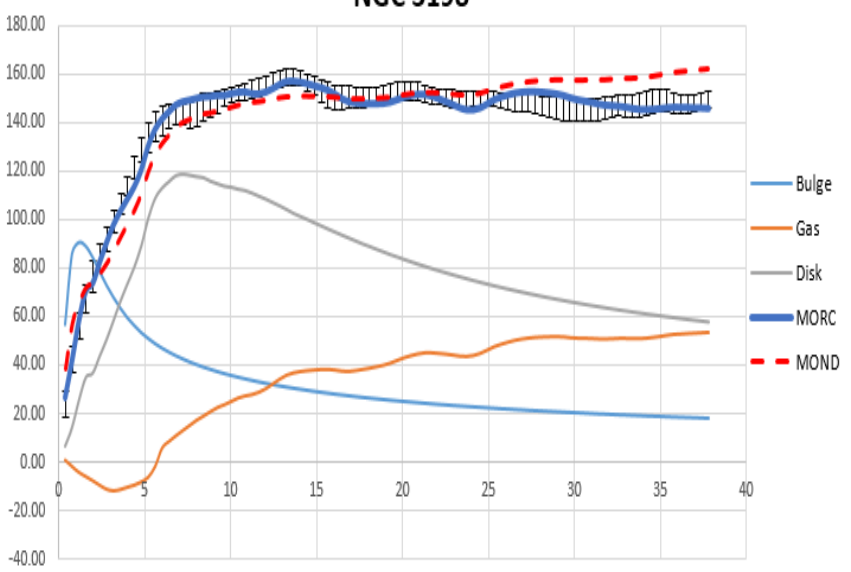

NGC 2403

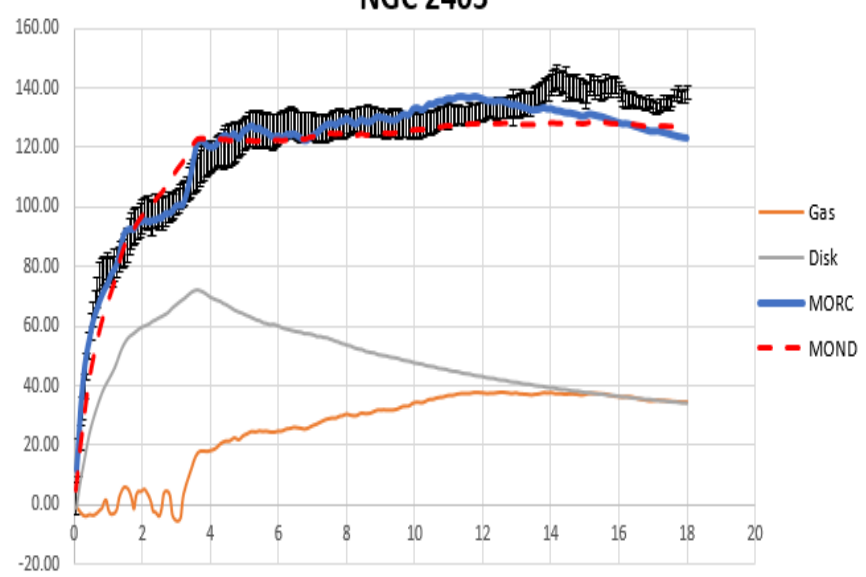

NGC 2976

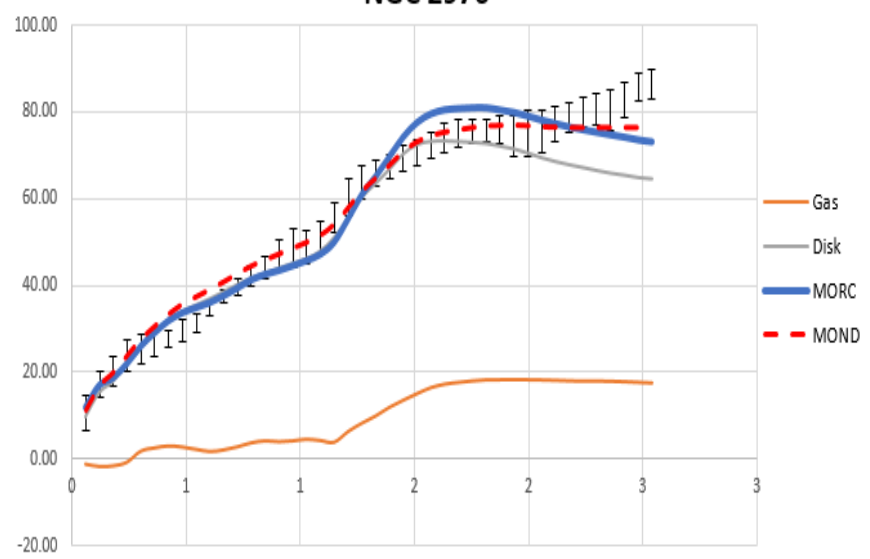

NGC 3621

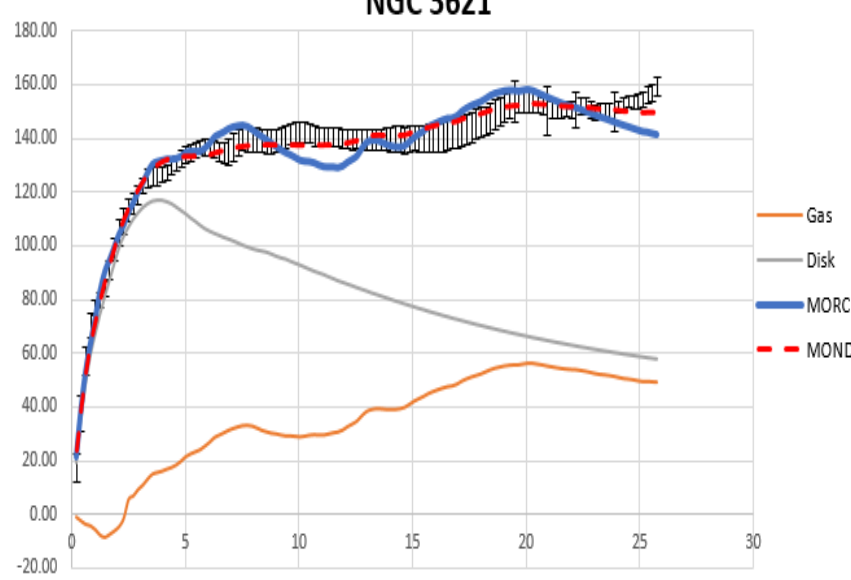

NGC 7793

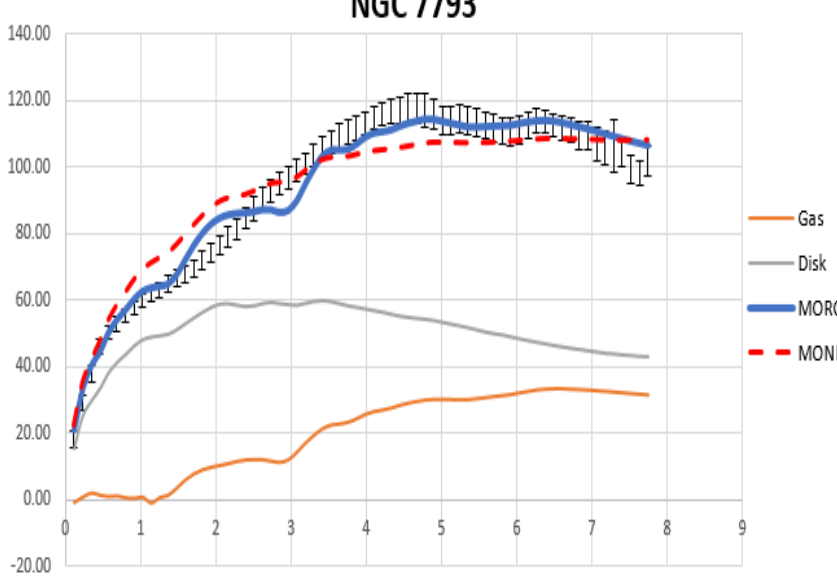

DD0 154

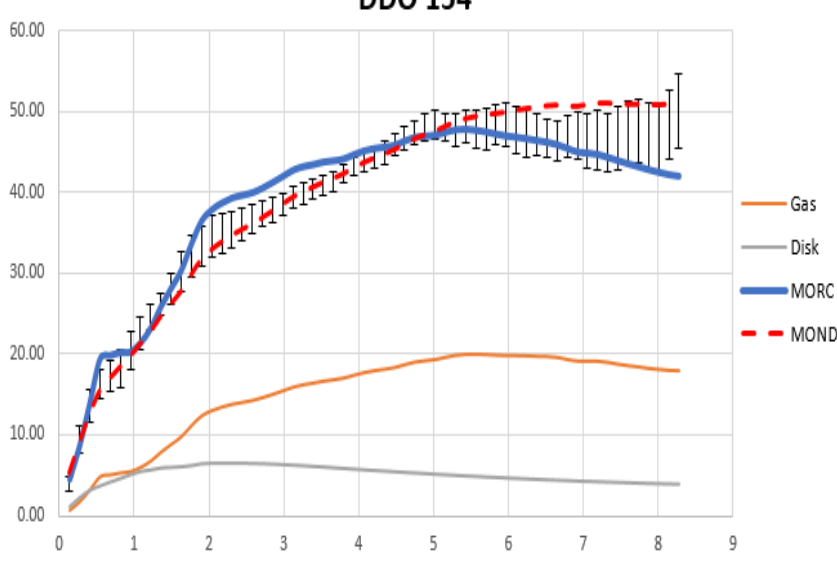

Figure 4. More graphs of our fits (solid) and MOND fits (dashed) of THINGS galactic RC's (with error bars). Our fit disappears when it lies right underneath MOND. Disk, gas and bulge curves are labeled. Bulge curves are not always available. Vertical axis is rotation velocity in $\mathrm{km} / \mathrm{s}$ and horizontal axis is orbital radius in kpc. Note: The MOND distance fitting factor $\gamma_{\text {dist }}$ would alter the horizontal scale proportionally. Corresponding numerical results are in Tables $1 \& 2$. 

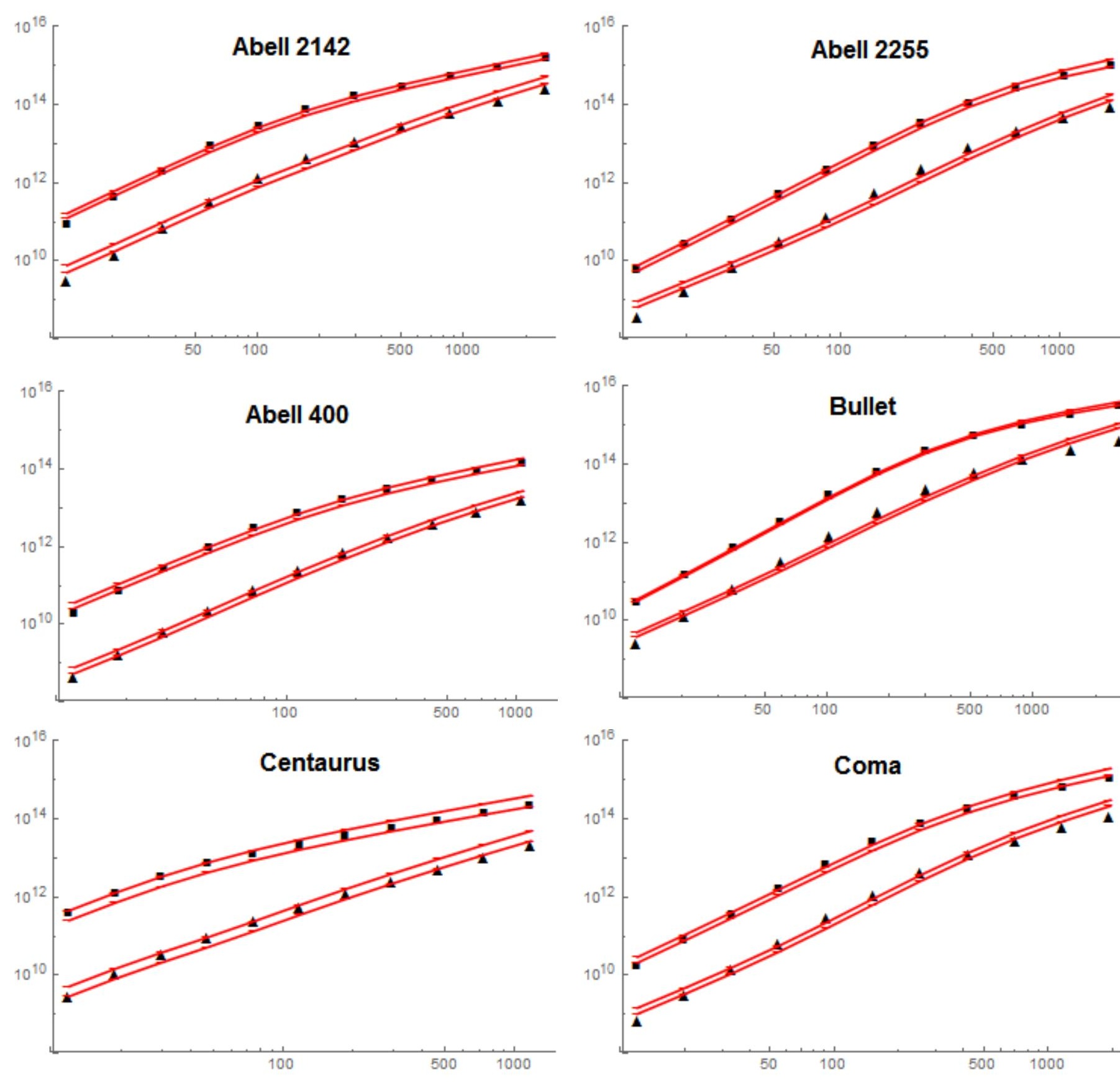

Figure 5. Log-log plots of our fits and MSTG fits of X-ray cluster mass profiles (compiled from ROSAT and ASCA data). Vertical scale is in solar masses and horizontal scale is in kpc. Our ansatz is increasing the gas mass (triangles) to fit the dynamical mass (squares). MSTG is decreasing the dynamical mass to fit the gas mass. The sizes of the objects are approximately equal to their errors. Our fit is the upper pair of lines (connecting fit points) over the squares where line separation corresponds to error. MSTG fit is the lower pair of lines (connecting fit points) over the triangles where line separation corresponds to error. Corresponding numerical results are in Tables $3 \& 4$. 

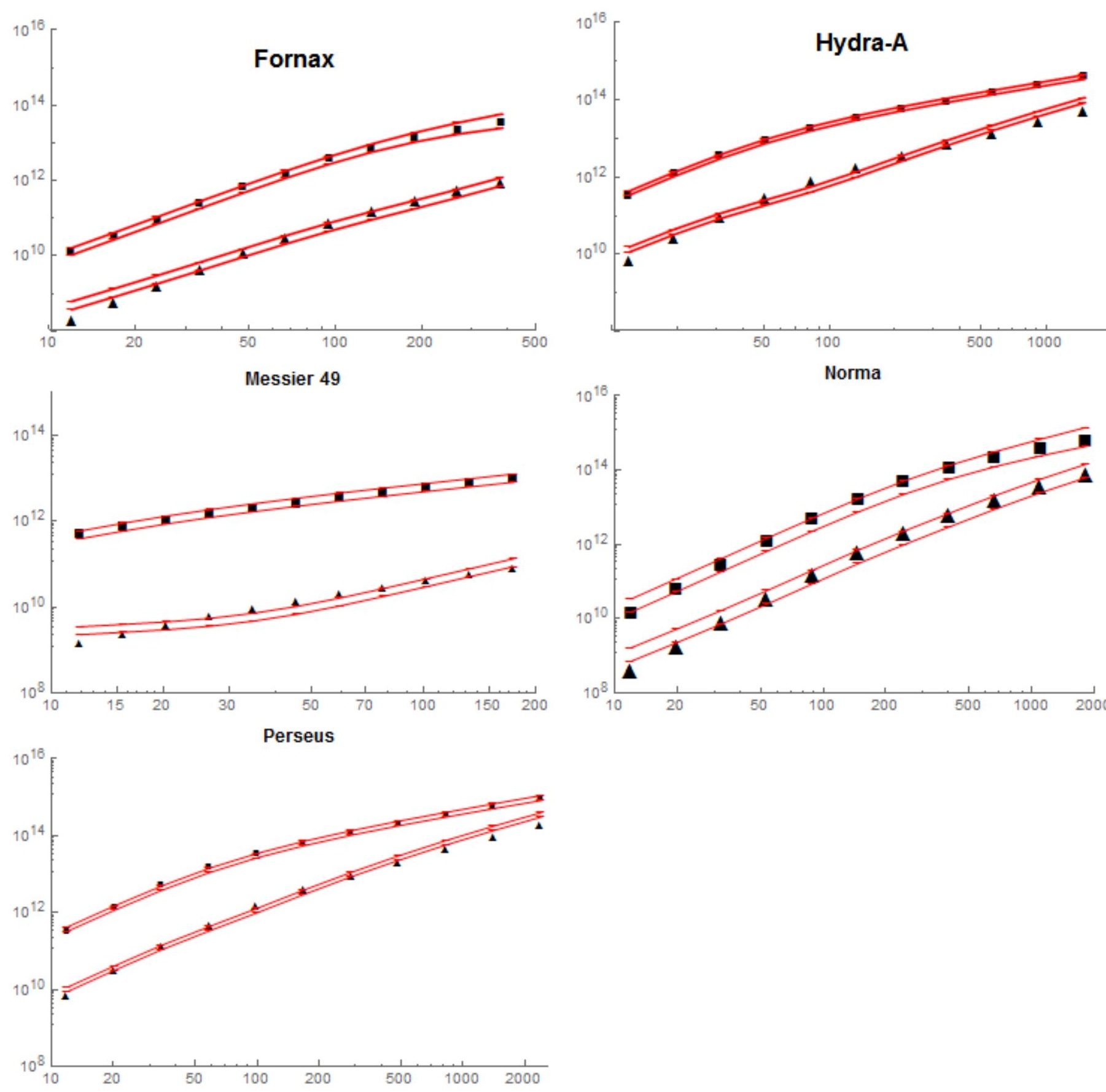

Figure 6. More log-log plots of our fits and MSTG fits of X-ray cluster mass profiles (compiled from ROSAT and ASCA data). Vertical scale is in solar masses and horizontal scale is in kpc. Our ansatz is increasing the gas mass (triangles) to fit the dynamical mass (squares). MSTG is decreasing the dynamical mass to fit the gas mass. The sizes of the objects are approximately equal to their errors. Our fit is the upper pair of lines (connecting fit points) over the squares where line separation corresponds to error. MSTG fit is the lower pair of lines (connecting fit points) over the triangles where line separation corresponds to error. Corresponding numerical results are in Tables $3 \& 4$. 
15. Blanchet, L., and Le Tiec, A.: Model of dark matter and dark energy based on gravitational polarization. Physical Review D 78, 024031 (2008).

16. Brownstein, J.R., and Moffat, J.W.: Galaxy Rotation Curves Without Non-Baryonic Dark Matter. The Astrophysical Journal 636, 721-741 (2006).

17. Brownstein, J.R., and Moffat, J.W.: Galaxy Cluster Masses Without Non-Baryonic Dark Matter. Monthly Notices of the Royal Astronomical Society 367, 527-540 (2006) http:/ / arxiv.org/abs/astro-ph/0507222

18. Moffat, J.W.: Gravitational Theory, Galaxy Rotation Curves and Cosmology without Dark Matter. Journal of Cosmology and Astroparticle Physics 2005, http:/ /dx.doi.org/10.1088/1475-7516/2005/05/003 (2005)

19. Moffat, J.W.: A Modified Gravity and its Consequences for the Solar System, Astrophysics and Cosmology. International Journal of Modern Physics D 16, 2075-2090 (2008) http:/ / arxiv.org/abs/gr-qc/0608074

20. Moffat, J.W.: Scalar-Tensor-Vector Gravity Theory. Journal of Cosmology and Astroparticle Physics 0603, 004 (2006) https:/ /arxiv.org/abs/gr-qc/0506021

21. Moffat, J.W., and Rahvar, S.: The MOG weak field approximation and observational test of galaxy rotation curves. Monthly Notices of the Royal Astronomical Society 436, 1439-1451 (2013) https://arxiv.org/abs/1306.6383

22. Moffat, J.W., and Rahvar, S.: The MOG Weak Field approximation II. Observational test of Chandra X-ray Clusters. Monthly Notices of the Royal Astronomical Society 441, 3724-3732 (2014) https: / / arxiv.org/abs/1309.5077

23. Israel, N.S., and Moffat, J..: The Train Wreck Cluster and Bullet Cluster explained by modified gravity without dark matter. https: / /arxiv.org/abs/1606.09128

24. Rodrigues, D.C., Piattella, O.F., Fabris, J.C., and Shapiro, I.L.: Renormalization Group approach to Gravity: the running of $G$ and $\Lambda$ inside galaxies and additional details on the elliptical NGC 4494. Proceedings of Science, VIII International Workshop on the Dark Side of the Universe (2012) https://arxiv.org/abs/1301.4148

25. Mashhoon, B.: Nonlocal General Relativity. Galaxies 3, 1-17 (2015) https:/ /arxiv.org/abs/1411.5411

26. Bergstrom, L.: Non-baryonic dark matter: observational evidence and detection methods. 2000 Reports on Progress in Physics 63, 793 doi:10.1088/0034-4885/63/5/2r3

27. Young, M.: No Dark Matter from LUX Experiment. Sky \& Telescope, 26 July 2016 http://www.skyandtelescope.com/astronomy-news/no-dark-matter-from-lux-experiment/

28. Gentile, G., Burkert, A., Salucci, P., Klein, U., and Walter, F.: The Dwarf Galaxy DDO 47 as a Dark Matter Laboratory: Testing Cusps Hiding in Triaxial Halos. The Astrophysical Journal Letters 634, doi:10.1086/498939 (2005) http:/ / arxiv.org/pdf/astro-ph/0506538.pdf

29. de Blok W.: The Core-Cusp Problem. Advances in Astronomy 2010, Article ID 789293, 14 pages, 2010. doi:10.1155/2010/789293

30. Clifton, T., Ferreira, P.G., Padilla, A., and Skordis, C.: Modified Gravity and Cosmology. Physics Reports 513(1), 1-189 (2012)

31. McGaugh, S.: A Tale of Two Paradigms: the Mutual Incommensurability of LCDM and MOND. Canadian Journal of Physics 93, 250 (2015) arXiv:1404.7525

32. Carroll, S.: Why Is There Dark Matter? (July 2015) http:/ / www.preposterousuniverse.com/blog/2015/07/07/why-is-there-dark-matter /

33. Bull, P., et al.: Beyond $\Lambda$ CDM: Problems, solutions, and the road ahead. http://arxiv.org/abs/1512.05356, p 67

34. Wald, R.: General Relativity. University of Chicago Press, Chicago (1984).

35. Stuckey, W.M.: The observable universe inside a black hole. American Journal of Physics 62, $788-795$ (1994).

36. Caravelli, F., and Markopoulou, F.: Disordered Locality and Lorentz Dispersion Relations: An Explicit Model of Quantum Foam. Physical Review D 86, 024019 (2012) http:/ /arxiv.org/pdf/1201.3206v1.pdf

37. Prescod-Weinstein, C., and Smolin, L.: Disordered Locality as an Explanation for the Dark Energy. Physical Review D 80, 063505 (2009) http:/ / arxiv.org/pdf/0903.5303.pdf

38. Regge, T.: General relativity without coordinates. Nuovo Cimento 19, 558-571 (1961)

39. Misner, C.W., Thorne, K.S., and Wheeler, J.A.: Gravitation. W.H. Freeman, San Francisco (1973), Chapter 42, p 1166.

40. Barrett, J.W.: The geometry of classical Regge calculus. Classical and Quantum Gravity 4, 1565-1576 (1987) 
41. Williams, R.M., and Tuckey, P.A.: Regge calculus: a brief review and bibliography. Classical and Quantum Gravity 9, 1409-1422 (1992)

42. Wheeler, J.A., and Feynman, R.P.: Classical Electrodynamics in Terms of Direct Interparticle Action. Reviews of Modern Physics 21, 425-433 (1949)

43. Hawking, S.W.: On the Hoyle-Narlikar theory of gravitation. Proceedings of the Royal Society of London. Series A, Mathematical and Physical Sciences 286, 313-320 (1965)

44. Davies, P.C.W.: Extension of Wheeler-Feynman quantum theory to the relativistic domain I. Scattering processes. Journal of Physics A: General Physics 4, 836-845 (1971)

45. Davies, P.C.W.: Extension of Wheeler-Feynman quantum theory to the relativistic domain II. Emission processes. Journal of Physics A: General Physics 5, 1025-1036 (1972)

46. Hoyle, F., and Narlikar, J.V.: Cosmology and action-at-a-distance electrodynamics. Reviews of Modern Physics 67, 113-155 (1995)

47. Narlikar, J.V.: Action at a Distance and Cosmology: A Historical Perspective. Annual Review of Astronomy and Astrophysics 41, 169-189 (2003)

48. Magalhaes, N.S., and Cooperstock, F.I.: Galactic mapping with general relativity and the observed rotation curves. (2015) http:/ /arxiv.org/abs/1508.07491

49. Carrick, J.D., and Cooperstock, F.I.: General relativistic dynamics applied to the rotation curves of galaxies. Astrophysics and Space Science 337, 321-329 (2012).

50. Cooperstock, F.I., and Tieu, S.: Galactic dynamics via general relativity - A compilation and new results. International Journal of Modern Physics A 13, 2293-2325 (2007).

51. McGaugh, S., Lelli, F., and Schombert, J.: The Radial Acceleration Relation in Rotationally Supported Galaxies. Accepted for publication in Physical Review Letters https:/ /arxiv.org/abs/1609.05917

52. Stuckey, W.M., McDevitt, T., and Silberstein, M.: Modified Regge Calculus as an Explanation of Dark Energy. Classical and Quantum Gravity 29, 055015 (2012a) http:/ /arxiv.org/abs/1110.3973

53. Stuckey, W.M., McDevitt, T., and Silberstein, M.: Explaining the Supernova Data without Accelerating Expansion. International Journal of Modern Physics D 21(11), 1242021 (2012b).

54. Stuckey, W.M., McDevitt, T., Sten, A.K., and Silberstein, M.: End of a Dark Age? International Journal of Modern Physics D 25(12) 1644004 (2016) https:/ /arxiv.org/abs/1605.09229

55. Hu, W.: Wandering in the Background: A Cosmic Microwave Background Explorer. PhD Thesis (1995) http://arxiv.org/abs/astro-ph/9508126

56. Teukolsky, S.: The Kerr Metric. Classical \& Quantum Gravity 32, 124006 (2015).

57. Brownstein, J.R.: Modified Gravity and the phantom of dark matter. PhD Thesis (2009) http://arxiv.org/abs/0908.0040

58. Wong, C.: Applications of Regge calculus to the Schwarzschild and Reissner-Nordstrom geometries at the moment of time symmetry. Journal of Mathematical Physics 12, 70-78 (1971).

59. Gentile, G., Famaey, B., and de Blok, W.: THINGS about MOND. Astronomy and Astrophysics A 76, 527-538 (2011) http:/ /arxiv.org/abs/0810.2125v1

60. Richtler, T., Schuberth, Y., Hilker, M., Dirsch, B., Bassino, L., and Romanowsky, A.: The dark matter halo of NGC 1399- CDM or MOND? Astronomy and Astrophysics 478, L23-L26. doi:10.1051/0004-6361:20078539 (2008).

61. Walter, F., Brinks, E., de Blok, W., Bigiel, F., Kennicutt, R., Thornley, M., and Leroy, A.: THINGS: The HI Nearby Galaxy Survey.The Astronomical Journal 136, doi:10.1088/0004-6256/136/6/2563 (2008) http:/ /arxiv.org/pdf/0810.2125.pdf

62. Reiprich, T.H: Cosmological Implications and Physical Properties of an X-Ray Flux-Limited Sample of Galaxy Clusters, PhD Thesis (2001)

63. Reiprich, T.H., and Böhringer, H.: The Mass Function of an X-Ray Flux-limited Sample of Galaxy Clusters. The Astrophysical Journal 567, 716-740 (2002)

64. Clowe, D., Bradac, M., Gonzalez, A., Markevitch, M., Randall, S., Jones, C., and Zaritsky, D.: A Direct Empirical Proof of the Existence of Dark Matter. The Astrophysical Journal Letters 648, L109-L113 (2006) http:/ /arxiv.org/abs/astro-ph/0608407

65. Brownstein, J.R., and Moffat, J.W.: The Bullet Cluster 1E0657-558 evidence shows Modified Gravity in the absence of Dark Matter. Monthly Notices of the Royal Astronomical Society 382, $29-47$ (2007) arXiv:astro-ph/0702146 
66. Carroll, S.: Dark Matter: Just Fine, Thanks.

http://www.preposterousuniverse.com/blog/2011/02/26/dark-matter-just-fine-thanks/ 26 February 2011

67. Skordis, C., Mota, D.F., Ferreira, P.G., and Boehm, C.: Large scale structure in Bekenstein's theory of relativistic Modified Newtonian Dynamics. Physical Review Letters 96, 011301 (2006) http:/ /arxiv.org/abs/astro-ph/0505519

68. Skordis, C.: The Tensor-Vector-Scalar theory and its cosmology. Classical \& Quantum Gravity 26, 143001 (2009) http://arxiv.org/abs/0903.3602

69. McGaugh, S.: A Tale of Two Paradigms: the Mutual Incommensurability of $\Lambda$ CDM and MOND. Canadian Journal of Physics 93(2), 250-259 (2015) http:/ / arxiv.org/abs/1404.7525

70. Skordis, C., Personal communication (Sep 2016)

71. Angus, G.: Are sterile neutrinos consistent with clusters, the CMB and MOND? Monthly Notices of the Royal Astronomical Society 394(1), 527-532 (2009) http:/ / arxiv.org/abs/0805.4014

72. McGaugh, S., Personal communication (Sep 2016)

73. Moffat, J.W., and Toth, V.: Cosmological Observations in a Modified Theory of Gravity (MOG), Galaxies 1(1), 65-82 (2013) http:/ /www.mdpi.com/2075-4434/1/1/65/htm

74. Mukhanov, V.: Physical Foundations of Cosmology, Cambridge University Press (2005), Chapter 9

75. Hu, W., and Sugiyama, N.: Anisotropies in the Cosmic Microwave Background: An Analytic Approach. Astrophysical Journal 444(2), Part 1, 489-506 (1995) https:/ / arxiv.org/abs/astro-ph/9407093

76. Hu, W., and Sugiyama, N.: Small Scale Cosmological Perturbations: An Analytic Approach. Astrophysical Journal 471, 542-570 (1996).

77. Planck Collaboration: Planck 2015 results. XIII. Cosmological parameters. Astronomy \& Astrophysics 594, A13 DOI:10.1051/0004-6361/201525830 (2016) https:/ /arxiv.org/abs/1502.01589

78. The file COM PowerSpect CMB R2.02.fits at http://irsa.ipac.caltech.edu/data/Planck/release 2/ancillary-data/HFI Products.html

79. The file COM PowerSpect CMB-base-plikHM-TT-lowTEB-minimum-theory R2.02.txt at http://pla.esac.esa.int/pla/

(C) 2016 by the authors; licensee Preprints, Basel, Switzerland. This article is an open access article distributed under the terms and conditions of the Creative Commons Attribution (CC-BY) license (http://creativecommons.org/licenses/by/4.0/). 